\title{
Gauge equivalence of Dirac structures and symplectic groupoids
}

\author{
Henrique Bursztyn* \\ Mathematical Sciences Research Institute \\ 1000 Centennial Drive, Berkeley \\ CA, 94720 \\ and \\ Olga Radko ${ }^{\dagger}$ \\ Department of Mathematics \\ University of California, Berkeley \\ CA, 94720
}

September, 2002

\begin{abstract}
We study gauge transformations of Dirac structures and the relationship between gauge and Morita equivalences of Poisson manifolds. We describe how the symplectic structure of a symplectic groupoid is affected by a gauge transformation of the Poisson structure on its identity section, and prove that gauge-equivalent integrable Poisson structures are Morita equivalent. As an example, we study certain generic sets of Poisson structures on Riemann surfaces: we find complete gauge-equivalence invariants for such structures which, on the 2-sphere, yield a complete invariant of Morita equivalence.
\end{abstract}

\section{Introduction}

Dirac structures were introduced in $[10,11]$ to provide a geometric framework for the study of constrained mechanical systems. Examples of Dirac structures on a manifold $M$ include presymplectic forms, Poisson structures and foliations; in general, a Dirac structure determines a singular foliation on $M$ whose leaves carry a pre-symplectic structure.

The notion of gauge equivalence of Dirac structures was introduced in [27] motivated by the study of the geometry of Poisson structures "twisted" by a closed 3-form. (See also [21, 24], where such structures were introduced in connection with Poisson-sigma models.) There is a natural way to modify a Dirac structure on $M$ by a closed 2-form $B \in \Omega^{2}(M)$ : one adds the pull-back of $B$ to the pre-symplectic form on each leaf of the foliation, and the resulting pre-symplectic foliation determines a new Dirac structure. This operation is called a gauge transformation, and it defines an action of the additive group of closed 2-forms on Dirac structures. Two Dirac

\footnotetext{
${ }^{*}$ Current address: Department of Mathematics, University of Toronto, Toronto, Ontario, M5S 3G3. E-mail: henrique@math.toronto.edu

${ }^{\dagger}$ E-mail: radko@math.berkeley.edu
} 
structures are called gauge equivalent if they lie in the same orbit of this action. As discussed in [27], gauge-equivalent Dirac structures share a lot of important properties; for instance, their corresponding Lie algebroids are always isomorphic.

Gauge transformations of Poisson structures also arise in some quantization problems. Let $(M, \pi)$ be a Poisson manifold, and let $\operatorname{Def}(M, \pi)$ denote its moduli space of equivalence classes of star products [1]. It follows from Kontsevich's formality theorem [22] that $\operatorname{Def}(M, \pi)$ is in bijection with equivalence classes of formal Poisson structures on $(M, \pi)$. As discussed in [4], the classification of deformation quantizations of $(M, \pi)$ up to Morita equivalence can be expressed in terms of the orbits of a canonical action of the $\operatorname{Picard} \operatorname{group} \operatorname{Pic}(M) \cong H^{2}(M, \mathbb{Z})$ on $\operatorname{Def}(M, \pi)$. Following Kontsevich's correspondence, there is a Poisson counterpart of algebraic Morita equivalence given by an $H^{2}(M, \mathbb{Z})$-action on formal Poisson structures, and the results in $[4,5,20]$ indicate that this action is given by gauge transformations. In this context, a natural question is how gauge equivalence relates to the geometric notion of Morita equivalence of Poisson manifolds [33]. (See [23] for other aspects of the relationship between algebraic and geometric Morita equivalence.)

In this paper we discuss the notion of gauge equivalence in the realm of symplectic dual pairs and symplectic groupoids, and study the relationship between the notions of gauge and Morita equivalence of Poisson structures.

The paper is organized as follows.

In Section 2 we recall some basic facts about Dirac structures and their functorial properties, and establish an equivariance property of Dirac maps with respect to gauge transformations.

In Section 3 we extend the usual notion of a symplectic dual pair [29] to deal with Dirac manifolds. This more general notion, called a pre-dual pair, arises naturally when one considers a gauge transformation of a Poisson manifold which is part of a symplectic dual pair. We show that, under natural regularity conditions, ordinary symplectic dual pairs are obtained as quotients of pre-dual pairs.

In Section 4 we study the effect of gauge transformations of an integrable Poisson manifold on the symplectic structure of its symplectic groupoid. We show that if $(G, \Omega)$ is a symplectic groupoid and $G_{0}$ is its identity section, endowed with its natural Poisson structure $\pi$, then a gauge transformation of $\pi$ by $B \in \Omega^{2}\left(G_{0}\right)$ changes the symplectic form on $G$ by

$$
\Omega \mapsto \Omega+\alpha^{*} B-\beta^{*} B
$$

where $\alpha$ and $\beta$ are the source and target maps, respectively.

Using this result, we show in Section 5 that two integrable gauge-equivalent Poisson structures are Morita equivalent. The converse is clearly not true, as Morita equivalent Poisson structures need not have the same leaf decomposition. We show that, in fact, the converse is not true even if we consider the possibility of gauge equivalence up to a Poisson diffeomorphism.

Finally, in Section 6, we study gauge and Morita equivalence of a certain generic set of Poisson structures on a compact connected oriented surface $\Sigma$. We consider the space $\mathscr{G}_{n}(\Sigma)$ of Poisson structures vanishing linearly on $n$ smooth disjoint curves on $\Sigma$ and show that two Poisson structures $\pi, \pi^{\prime} \in \mathscr{G}_{n}(\Sigma)$ vanishing on the same curves are gauge equivalent if and only if they have the same corresponding modular periods. (A classification of these structures up to isomorphism was obtained in [25].) This result provides a sufficient condition for Morita equivalence in $\mathscr{G}_{n}(\Sigma)$; for the case $\Sigma=S^{2}$, we construct a complete Morita-equivalence invariant for such Poisson structures.

Acknowledgments: We would like to thank Robert Bryant, Marius Crainic, J.-P. Dufour, Rui L. Fernandes, André Henriques, Nicholas Proudfoot and Alan Weinstein for valuable discussions 
and comments. We also thank the referee for comments and corrections.

\section{Dirac structures}

\subsection{Linear Dirac structures}

Let $V$ be a finite-dimensional real vector space. Let us equip $V \oplus V^{*}$ with the symmetric pairing

$$
\langle(x, \omega),(y, \mu)\rangle=\frac{1}{2}(\omega(y)+\mu(x)) .
$$

A Dirac structure on $V$ is a subspace $L \subset V \oplus V^{*}$ which is maximally isotropic with respect to $\langle,\rangle^{1}$. As we will see later, it is useful to think of $\left(V \oplus V^{*},\langle\rangle,\right)$ as an "odd" symplectic vector space (in the sense of super geometry), in such a way that its lagrangian subspaces correspond to Dirac structures on $V$. We denote the set of Dirac structures on $V$ by $\operatorname{Dir}(V)$.

Throughout the text, we will identify bilinear forms $\Omega: V \times V \longrightarrow \mathbb{R}\left(\right.$ resp. $\left.\pi: V^{*} \times V^{*} \longrightarrow \mathbb{R}\right)$ with linear maps $\tilde{\Omega}: V \longrightarrow V^{*}$ (resp. $\left.\tilde{\pi}: V^{*} \longrightarrow V\right)$ by $\widetilde{\Omega}(u)(v)=\Omega(u, v)(\operatorname{resp} . \tilde{\pi}(\nu)(\eta)=$ $\pi(\eta, \nu))$. Whenever the context is clear, we will just write $\Omega$ for $\widetilde{\Omega}(\operatorname{resp} . \pi$ for $\tilde{\pi}$ ).

Example 2.1 If $\Omega$ is a skew-symmetric bilinear form on $V$ (resp. $\pi$ is a skew-symmetric bilinear form on $V^{*}$, i.e., a bivector on $\left.V\right)$, then $L=\operatorname{graph}(\Omega) \subset V \oplus V^{*}(\operatorname{resp} . L=\operatorname{graph}(\pi))$ is a Dirac structure on $V$. Conversely, any Dirac structure $L$ satisfying $L \cap V^{*}=\{0\}$ (resp. $L \cap V=\{0\}$ ) defines a skew-symmetric bilinear form (resp. bivector) on $V$. Hence pre-symplectic structures (i.e., skew-symmetric bilinear forms) and Poisson structures (i.e., bivectors) on vector spaces are examples of linear Dirac structures.

Consider the natural projections $\rho: V \oplus V^{*} \longrightarrow V$, and $\rho^{*}: V \oplus V^{*} \longrightarrow V^{*}$, and let $L$ be a Dirac structure on $V$. It is easy to check that [10]

$$
\begin{aligned}
& \rho(L)^{\circ}=L \cap V^{*}, \\
& \rho^{*}(L)^{\circ}=L \cap V,
\end{aligned}
$$

where ${ }^{\circ}$ denotes the annihilator.

Proposition 2.2 A Dirac structure $L$ on $V$ is equivalent to either of the following:

i) A pair $(R, \Omega)$, where $R \subseteq V$ is a subspace and $\Omega$ is a skew-symmetric bilinear form on $R$.

ii) A pair $(K, \pi)$, where $K \subseteq V$ is a subspace and $\pi$ is a bivector on the quotient $V / K$.

Moreover, these correspondences are such that $R=\rho(L)$ and $K=V \cap L=\operatorname{ker} \Omega$.

Proof: Let $L$ be a Dirac structure on $V$. We set $R=\rho(L) \subseteq V$ and define the skew-symmetric bilinear form $\Omega_{L}: R \longrightarrow R^{*}$ by

$$
\left.\Omega_{L}(x) \doteq \eta\right|_{R},
$$

where $\eta \in V^{*}$ is any element such that $(x, \eta) \in L$. The map $\Omega_{L}$ is well defined by $(2.2)$, and $\operatorname{ker} \Omega_{L}=V \cap L$. Similarly, we can define a skew-symmetric form $\pi_{L}$ on $\rho^{*}(L)$ and notice that $\left(\rho^{*}(L)\right)^{*}=V / \rho^{*}(L)^{\circ}=V / V \cap L$. So, for $K \doteq V \cap L$, we have a well-defined bivector $\pi_{L}$ on $V / K$ with $\operatorname{ker} \pi_{L}=L \cap V^{*}$.

\footnotetext{
${ }^{1}$ This is equivalent to $L$ being isotropic and $\operatorname{dim} L=\operatorname{dim} V$.
} 
Conversely, given a pair $(R, \Omega)$ as in $i)$, we set

$$
L \doteq\left\{(x, \eta) \mid x \in R, \eta \in V^{*} \text { such that }\left.\eta\right|_{R}=\Omega(x)\right\} .
$$

A simple dimension count shows that $L$ is a Dirac structure satisfying $\rho(L)=R$ and $\Omega_{L}=\Omega$. A similar construction holds for a pair $(K, \pi)$ as in $i i)$.

For $L \in \operatorname{Dir}(V)$, we denote the corresponding skew-symmetric bilinear form on $\rho(L)$ by $\Omega_{L}$ and the corresponding bivector on $V / V \cap L$ by $\pi_{L}$.

Example 2.3 Let $L=\operatorname{graph}(\Omega)$, where $\Omega$ is a skew-symmetric bilinear form on $V$. Then $R=\rho(L)=V, \Omega_{L}=\Omega, K=\operatorname{ker} \Omega$, and $\pi_{L}$ is the Poisson bivector on the reduced space $V / K$ corresponding to the symplectic form induced by $\Omega$.

Example 2.4 Let $L=\operatorname{graph}(\pi)$, where $\pi$ is a bivector on $V$. Then $R=\pi\left(V^{*}\right)$, and $\Omega_{L}$ is the natural symplectic form induced by $\pi$. In this case, $K=\operatorname{ker} \Omega_{L}=\{0\}$, and $\pi_{L}=\pi$.

\subsection{Functorial properties of linear Dirac structures}

Linear Dirac structures have nice functorial properties: they can be both pushed forward and pulled back. This is a consequence of the dual characterizations of Dirac structures (Prop. 2.2) in terms of bilinear forms and bivectors.

We can also describe the functorial properties of Dirac structures in terms of Weinstein's symplectic category [28], where the objects are symplectic vector spaces and the morphisms are canonical relations. We recall here the main ideas.

Let $E, F$ and $H$ be symplectic vector spaces. A canonical relation between $E$ and $F$ is a lagrangian subspace $L \subset E \times \bar{F}$, where $\bar{F}$ is the vector space $F$ with symplectic form multiplied by -1 . If $L_{1} \subset E \times \bar{F}$ and $L_{2} \subset F \times \bar{H}$, the usual composition of relations

$$
L_{1} \circ L_{2}=\left\{(x, y) \in E \times H \mid \exists z \in F \text { such that }(x, z) \in L_{1} \text { and }(z, y) \in L_{2}\right\}
$$

defines a lagrangian subspace of $E \times \bar{H}$. If $L_{1}=\operatorname{graph}(f), L_{2}=\operatorname{graph}(g)$ for symplectomorphisms $f: F \longrightarrow E$ and $g: H \longrightarrow F$, then $L_{1} \circ L_{2}=\operatorname{graph}(f \circ g)$. In general, the composition of canonical relations defines a map

$$
\circ: \operatorname{Lag}(E \times \bar{F}) \times \operatorname{Lag}(F \times \bar{H}) \longrightarrow \operatorname{Lag}(E \times \bar{H}),
$$

where $\operatorname{Lag}(U)$ denotes the set of lagrangian subspaces of a vector space $U$. We remark that the same ideas work when symplectic vector spaces are replaced by vector spaces equipped with a nondegenerate symmetric bilinear form with zero signature (thought of as "odd" symplectic spaces); in this case, lagrangian (i.e., maximally isotropic) subspaces still have half the dimension of the total space.

Let $V$ and $W$ be vector spaces, and let $\phi: V \longrightarrow W$ be a linear map. Let $E=\left(V \oplus V^{*},\langle\rangle,\right)$ and $F=\left(W \oplus W^{*},\langle\rangle,\right)$, regarded as "odd" symplectic vector spaces. We define two canonical relations associated to $\phi$ :

$$
\begin{aligned}
& \mathcal{F} \phi=\left\{\left(\phi(x), \eta, x, \phi^{*} \eta\right) \mid x \in V, \eta \in W^{*}\right\} \in \operatorname{Lag}(F \times \bar{E}), \\
& \mathcal{B} \phi=\left\{\left(x, \phi^{*} \eta, \phi(x), \eta\right) \mid x \in V, \eta \in W^{*}\right\} \in \operatorname{Lag}(E \times \bar{F}) .
\end{aligned}
$$


(As will become clear below, the letters $\mathcal{F}$ and $\mathcal{B}$ stand for forward and backward.)

Since we have the natural identifications $\operatorname{Dir}(V) \cong \operatorname{Lag}(E \times\{0\})$ and $\operatorname{Dir}(W) \cong \operatorname{Lag}(F \times\{0\})$, the composition of relations (2.7) immediately induces maps

$$
\begin{aligned}
& \mathcal{F} \phi: \operatorname{Dir}(V) \longrightarrow \operatorname{Dir}(W), \\
& \mathcal{B} \phi: \operatorname{Dir}(W) \longrightarrow \operatorname{Dir}(V) .
\end{aligned}
$$

Explicitly, for $L_{V} \in \operatorname{Dir}(V), L_{W} \in \operatorname{Dir}(W)$ we have

$$
\begin{aligned}
& \mathcal{F} \phi\left(L_{V}\right)=\left\{(\phi(x), \eta) \mid x \in V, \eta \in W^{*},\left(x, \phi^{*} \eta\right) \in L_{V}\right\}, \\
& \mathcal{B} \phi\left(L_{W}\right)=\left\{\left(x, \phi^{*} \eta\right) \mid x \in V, \eta \in W^{*},(\phi(x), \eta) \in L_{W}\right\} .
\end{aligned}
$$

Example 2.5 If $L_{V}=\operatorname{graph}(\pi)$ for a bivector $\pi$ on $V$, then $\mathcal{F} \phi\left(L_{V}\right)=\operatorname{graph}\left(\phi_{*} \pi\right)$. Analogously, if $L_{W}=\operatorname{graph}(\Omega)$ for a skew-symmetric bilinear form $\Omega$ in $W$, then $\mathcal{B} \phi\left(L_{W}\right)=\operatorname{graph}\left(\phi^{*} \Omega\right)$.

We observe that the maps $\mathcal{F} \phi$ and $\mathcal{B} \phi$ are not inverse to each other in general. A simple computation shows that if $\phi$ is $1-1$, then $\mathcal{B} \phi \circ \mathcal{F} \phi=\operatorname{Id}$, and if $\phi$ is onto, then $\mathcal{F} \phi \circ \mathcal{B} \phi=\operatorname{Id}$.

Proposition 2.6 Let $\phi: V \longrightarrow W$ be a linear map, and let $L_{V} \in \operatorname{Dir}(V)$ and $L_{W} \in \operatorname{Dir}(W)$.

i) If $\mathcal{F} \phi\left(L_{V}\right)=L_{W}$, then $\operatorname{ker} \Omega_{L_{W}}=\phi\left(\operatorname{ker} \Omega_{L_{V}}\right)$ and $\pi_{L_{W}}=\phi_{*}\left(\pi_{L_{V}}\right)$.

ii) If $L_{V}=\mathcal{B} \phi\left(L_{W}\right)$, then $\rho\left(L_{V}\right)=\phi^{-1}\left(\rho\left(L_{W}\right)\right)$ and $\Omega_{L_{V}}=\phi^{*}\left(\Omega_{L_{W}}\right)$.

Proof: Using (2.12), it is easy to check that if $L_{W}=\mathcal{F} \phi\left(L_{V}\right)$, then $\operatorname{ker} \Omega_{L_{W}}=W \cap L_{W}=$ $\left\{\phi(x) \mid x \in V,(x, 0) \in L_{V}\right\}$. Since $V \cap L_{V}=\operatorname{ker} \Omega_{L_{V}}$, it follows that $\operatorname{ker} \Omega_{L_{W}}=\phi\left(\operatorname{ker} \Omega_{L_{V}}\right)$.

As $\eta \in \phi\left(V \cap L_{V}\right)^{\circ}$ implies that $\phi^{*} \eta \in V \cap L_{V}$, we can define $\phi_{*} \pi_{L_{V}}$ on $W / \phi\left(V \cap L_{V}\right)$ by $\phi_{*} \pi_{L_{V}}(\eta)=\phi\left(\pi_{L_{V}}\left(\phi^{*} \eta\right)\right)$, where $\eta \in\left(W / \phi\left(V \cap L_{V}\right)\right)^{*} \cong W^{*} / \phi\left(V \cap L_{V}\right)^{\circ}$.

By definition of $\pi_{L_{V}}$ (see Prop. 2.2), $\phi\left(\pi_{L_{V}}\left(\phi^{*} \eta\right)\right)=\phi(x)$, where $x$ is such that $\left(x, \phi^{*} \eta\right) \in L_{V}$. On the other hand, if $y=\pi_{L_{W}}(\eta)$, then $(y, \eta) \in L_{W}=\mathcal{F} \phi\left(L_{V}\right)$, which is the case if and only if $y=\phi(x)$ and $\left(x, \phi^{*} \eta\right) \in L$. Therefore $\pi_{L_{W}}=\phi_{*}\left(\pi_{L_{V}}\right)$ and $\left.i\right)$ is proven.

The proof of $i i)$ is analogous and is left for the reader.

Corollary 2.7 Let $\left(V, \pi_{1}\right)$ and $\left(W, \pi_{2}\right)$ be Poisson vector spaces, and let $L_{\pi_{i}}=\operatorname{graph}\left(\pi_{i}\right), i=$ 1,2. A linear map $\phi: V \longrightarrow W$ is Poisson (i.e., $\varphi_{*} \pi_{1}=\pi_{2}$ ) if and only if $\mathcal{F} \phi\left(L_{\pi_{1}}\right)=L_{\pi_{2}}$.

Similarly, if $\left(V, \Omega_{1}\right)$ and $\left(W, \Omega_{2}\right)$ are pre-symplectic vector spaces, then a linear map $\phi: V \longrightarrow$ $W$ satisfies $\phi^{*} \Omega_{2}=\Omega_{1}$ if and only if $\mathcal{B} \phi\left(L_{\Omega_{2}}\right)=L_{\Omega_{1}}$, where $L_{\Omega_{i}}=\operatorname{graph}\left(\Omega_{i}\right), i=1,2$.

This motivates the following definition.

Definition 2.8 Let $V$ and $W$ be vector spaces endowed with Dirac structures $L_{V}, L_{W}$, respectively. A linear map $\phi: V \longrightarrow W$ is called forward Dirac if $\mathcal{F} \phi\left(L_{V}\right)=L_{W}$.

A map satisfying the analogous definition for $\mathcal{B} \phi$ is called backward Dirac. We remark that these definitions are not equivalent. In this paper we will only deal with forward Dirac maps, and we will refer to them simply as Dirac maps.

Example 2.9 Let $L \in \operatorname{Dir}(V)$. As observed in Prop. 2.2(ii), $V /$ ker $\Omega_{L}$ has an induced Poisson structure $\pi_{L}$. The projection pr: $V \longrightarrow V / \operatorname{ker} \Omega_{L}$ is a Dirac map. 
Lemma 2.10 Let $L_{V} \in \operatorname{Dir}(V)$ and $L_{W} \in \operatorname{Dir}(W)$. If $\Phi: V \longrightarrow W$ is a Dirac map, then it naturally induces a Poisson map $\phi: V / \operatorname{ker} \Omega_{L_{V}} \longrightarrow W / \operatorname{ker} \Omega_{L_{W}}$.

Proof: Let $\operatorname{pr}_{V}: V \longrightarrow V / \operatorname{ker} \Omega_{L_{V}}$ and $\operatorname{pr}_{W}: W \longrightarrow W / \operatorname{ker} \Omega_{L_{W}}$ be the natural projections. By Prop. 2.2(i), the map $\phi: V / \operatorname{ker} \Omega_{L_{V}} \rightarrow W / \operatorname{ker} \Omega_{L_{W}}$ given by $\phi\left(\operatorname{pr}_{V}(x)\right) \doteq \operatorname{pr}_{W}(\Phi(x))$ is well defined. By the observation in Example 2.9, we get

$$
\mathcal{F} \phi\left(L_{\pi_{L_{V}}}\right)=\mathcal{F} \phi\left(\mathcal{F} \operatorname{pr}_{V}\left(L_{V}\right)\right)=\mathcal{F} \operatorname{pr}_{W}\left(\mathcal{F} \Phi\left(L_{V}\right)\right)=\mathcal{F} \operatorname{pr}_{W}\left(L_{W}\right)=L_{\pi_{L_{W}}},
$$

where $L_{\pi_{L_{V}}}=\operatorname{graph}\left(\pi_{L_{V}}\right), L_{\pi_{L_{W}}}=\operatorname{graph}\left(\pi_{L_{W}}\right)$ for the Poisson structures $\pi_{L_{V}}$ and $\pi_{L_{W}}$ on $V / \operatorname{ker} \Omega_{L_{V}}$ and $W / \operatorname{ker} \Omega_{L_{W}}$, respectively. Therefore, $\phi$ is a Poisson map.

\subsection{Gauge equivalence of linear Dirac structures}

Let $\operatorname{Bil}(W)$ be the additive group of skew-symmetric bilinear forms on a vector space $W$. Following Weinstein and Severa [27], we consider the action $\tau: \operatorname{Bil}(W) \times \operatorname{Dir}(W) \longrightarrow \operatorname{Dir}(W)$ by gauge transformations,

$$
\tau_{B}(L)=\{(x, \eta+B(x)) \mid(x, \eta) \in L\}, \quad B \in \operatorname{Bil}(W) .
$$

Alternatively, using Prop. 2.2, two Dirac structures $L_{1}, L_{2}$ on $W$ are gauge equivalent if

$$
\rho\left(L_{1}\right)=\rho\left(L_{2}\right) \text { and } \Omega_{L_{1}}=\Omega_{L_{2}}+\left.B\right|_{\rho\left(L_{2}\right)},
$$

for $B \in \operatorname{Bil}(W)$ and $L \in \operatorname{Dir}(W)$.

A linear map $\phi: V \longrightarrow W$ of vector spaces induces an action of $\operatorname{Bil}(W)$ on $\operatorname{Dir}(V)$, since $\phi^{*} \operatorname{Bil}(W) \subseteq \operatorname{Bil}(V)$.

Lemma 2.11 The map $\mathcal{F} \phi: \operatorname{Dir}(V) \longrightarrow \operatorname{Dir}(W)$ is $\operatorname{Bil}(W)$-equivariant.

Proof: We must show that $\mathcal{F} \phi\left(\tau_{\phi^{*} B}(L)\right)=\tau_{B}(\mathcal{F} \phi(L))$ for all $L \in \operatorname{Dir}(V), B \in \operatorname{Bil}(W)$.

Since $\tau_{\phi^{*} B}(L)=\left\{\left(x, \eta+\left(\phi^{*} B\right)(x)\right) \mid(x, \eta) \in L\right\}$, we have

$$
\mathcal{F} \phi\left(\tau_{\phi^{*} B}(L)\right)=\left\{(\phi(x), \xi) \mid\left(x, \phi^{*} \xi\right) \in \tau_{\phi^{*} B}(L)\right\}=\left\{(\phi(x), \xi) \mid\left(x, \phi^{*} \xi-\left(\phi^{*} B\right)(x)\right) \in L\right\} .
$$

On the other hand,

$$
\tau_{B}(\mathcal{F} \phi(L))=\left\{(\phi(x), \zeta+B(\phi(x))) \mid\left(x, \phi^{*} \zeta\right) \in L\right\}=\left\{(\phi(x), \xi) \mid\left(x, \phi^{*} \xi-\phi^{*}(B(\phi(x)))\right) \in L\right\} .
$$

Since $\left(\phi^{*} B\right)(x)=\phi^{*}(B(\phi(x)))$, the result follows.

A similar equivariance property holds for the map $\mathcal{B} \phi$.

Let $(V, \Omega)$ be a symplectic vector space, and let $(W, \pi)$ be a Poisson vector space. Let $L_{\Omega}=$ $\operatorname{graph}(\Omega)$ and $L_{\pi}=\operatorname{graph}(\pi)$ be the corresponding Dirac structures.

Lemma 2.12 Let $\phi:(V, \Omega) \longrightarrow(W, \pi)$ be a linear Poisson map, and let $B \in \operatorname{Bil}(W)$. Then the restricted map $\phi: \operatorname{ker}\left(\Omega+\phi^{*} B\right) \longrightarrow \operatorname{ker} \Omega_{\tau_{B}\left(L_{\pi}\right)}$ is an isomorphism. In particular, the form $\Omega+\phi^{*} B$ is symplectic if and only if $\tau_{B}\left(L_{\pi}\right)$ corresponds to a Poisson structure. 
Proof: By Lemma 2.11, $\mathcal{F} \phi\left(\tau_{\phi^{*} B}\left(L_{\Omega}\right)\right)=\tau_{B}\left(\mathcal{F} \phi\left(L_{\Omega}\right)\right)=\tau_{B}\left(L_{\pi}\right)$, since $\phi$ is Poisson. Hence, by Prop. $2.6(i)$,

$$
\operatorname{ker} \Omega_{\tau_{B}\left(L_{\pi}\right)}=\phi\left(\operatorname{ker}\left(\Omega+\phi^{*} B\right)\right),
$$

and $\phi: \operatorname{ker}\left(\Omega+\phi^{*} B\right) \longrightarrow \operatorname{ker} \Omega_{\tau_{B}\left(L_{\pi}\right)}$ is onto.

On the other hand, since $\left.\phi^{*} B\right|_{\operatorname{ker} \phi}=0$, it follows that

$$
\operatorname{ker}\left(\left.\phi\right|_{\operatorname{ker}\left(\Omega+\phi^{*} B\right)}\right)=\operatorname{ker} \phi \cap \operatorname{ker}\left(\Omega+\phi^{*} B\right)=\operatorname{ker} \phi \cap \operatorname{ker} \Omega=0 .
$$

So $\phi$ is injective.

In order to study dual pairs, it will be useful to collect a few results on pre-symplectic orthogonals in the linear case.

Lemma 2.13 Let $L \in \operatorname{Dir}(V)$ and $\phi: V \longrightarrow W$ be linear. Then

$$
(\operatorname{ker} \phi \cap \rho(L))^{\Omega_{L}}=\left\{x \in V \mid \exists \eta \in W^{*} \text { such that }\left(x, \phi^{*} \eta\right) \in L\right\} \text {. }
$$

Proof: If $x \in(\operatorname{ker} \phi \cap \rho(L))^{\Omega_{L}}$, then $\Omega_{L}(x)(y)=0$ for all $y \in \operatorname{ker} \phi \cap \rho(L)$. The form $\eta \in(\phi(\rho(L)))^{*}$ given by $\eta(\phi(y))=\Omega_{L}(x)(y)$ is well defined, $\phi^{*} \eta=\Omega_{L}(x)$, and hence $\left(x, \phi^{*} \eta\right) \in L$. On the other hand, suppose $\left(x, \phi^{*} \eta\right) \in L$ for $\eta \in W^{*}$. Then $\Omega_{L}(x)=\left.\phi^{*} \eta\right|_{\rho(L)}$, and if $y \in \operatorname{ker} \phi \cap \rho(L)$, we have $\Omega_{L}(x)(y)=\eta(\phi(y))=0$.

Let $L \in \operatorname{Dir}(V), L_{i} \in \operatorname{Dir}\left(W_{i}\right)$, and let $J_{i}: V \longrightarrow W_{i}, i=1,2$, be Dirac maps.

Lemma 2.14 Suppose that the following orthogonality condition on the $J_{i}$-fibers holds:

$$
\left(\text { ker } J_{1} \cap \rho(L)\right)^{\Omega_{L}}=\operatorname{ker} J_{2} \cap \rho(L) \text {. }
$$

Then for any $B \in \operatorname{Bil}\left(W_{2}\right), J_{1}:\left(V, \tau_{J_{2}^{*} B}(L)\right) \longrightarrow\left(W_{1}, L_{1}\right)$ is a Dirac map.

Proof: We must show that $\mathcal{F} J_{1}\left(\tau_{J_{2}^{*} B}(L)\right)=L_{1}$. Since these subspaces have the same dimension, it suffices to prove that $\mathcal{F} J_{1}(L)=L_{1} \subseteq \mathcal{F} J_{1}\left(\tau_{J_{2}^{*} B}(L)\right)$. Recall that

$$
\begin{aligned}
\mathcal{F} J_{1}\left(\tau_{J_{2}^{*} B}(L)\right) & =\left\{\left(J_{1}(x), \eta\right) \mid x \in V, \eta \in W_{1}^{*},\left(x, J_{1}^{*} \eta\right) \in \tau_{J_{2}^{*} B}(L)\right\} \\
& =\left\{\left(J_{1}(x), \eta\right) \mid x \in V, \eta \in W_{1}^{*},\left(x, J_{1}^{*} \eta-\left(J_{2}^{*} B\right)(x)\right) \in L\right\},
\end{aligned}
$$

and

$$
\mathcal{F} J_{1}(L)=\left\{\left(J_{1}(x), \eta\right) \mid x \in V, \eta \in W_{1}^{*},\left(x, J_{1}^{*} \eta\right) \in L\right\} .
$$

Suppose $\left(J_{1}(x), \eta\right) \in \mathcal{F} J_{1}(L)$. By Lemma 2.13, $x \in\left(\operatorname{ker} J_{1} \cap \rho(L)\right)^{\Omega_{L}}=\operatorname{ker} J_{2} \cap \rho(L)$. So $\left(J_{2}^{*} B\right)(x)=0$, and therefore $\left(x, J_{1}^{*} \eta-\left(J_{2}^{*} B\right)(x)\right)=\left(x, J_{1}^{*} \eta\right) \in L$. This implies that $\left(J_{1}(x), \eta\right) \in$ $\mathcal{F} J_{1}\left(\tau_{J_{2}^{*} B}(L)\right)$, and the result follows.

Consider a symplectic vector space $(V, \Omega)$, Poisson vector spaces $\left(W_{i}, \pi_{i}\right), i=1,2$, and linear Poisson maps $J_{i}: V \longrightarrow W_{i}$. We call the diagram

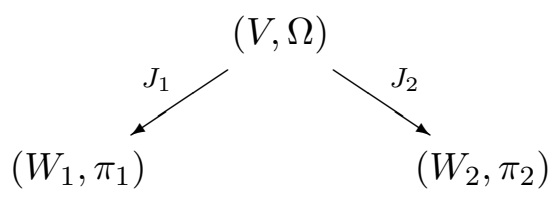


a linear dual pair if $\left(\operatorname{ker} J_{1}\right)^{\Omega}=\operatorname{ker} J_{2}$. Let us fix such a linear dual pair, and let $L_{\Omega}=\operatorname{graph}(\Omega)$ and $L_{\pi_{i}}=\operatorname{graph}\left(\pi_{i}\right)$. Before we prove the main result of this section, we need the following lemma.

Lemma 2.15 Let $B \in \operatorname{Bil}\left(W_{2}\right)$, and let $\Omega^{\prime}=\Omega+J_{2}^{*} B$. Then $\left(\operatorname{ker} J_{2}\right)^{\Omega^{\prime}}=\left(\operatorname{ker} J_{2}\right)^{\Omega}=\operatorname{ker} J_{1}$ and $\left(\operatorname{ker} J_{1}\right)^{\Omega^{\prime}}=\left(\operatorname{ker} J_{1}\right)^{\Omega}+\operatorname{ker} \Omega^{\prime}$.

Proof: Since $\left.J_{2}^{*} B\right|_{\text {ker } J_{2}}=0$, it follows that $\left(\operatorname{ker} J_{2}\right)^{\Omega^{\prime}}=\left(\operatorname{ker} J_{2}\right)^{\Omega}=\operatorname{ker} J_{1}$. By taking $\Omega^{\prime}$ orthogonals, we get $\left(\operatorname{ker} J_{1}\right)^{\Omega^{\prime}}=\operatorname{ker} J_{2}+\operatorname{ker} \Omega^{\prime}$.

We can now prove the main result of this section.

Theorem 2.16 Let $B_{i} \in \operatorname{Bil}\left(W_{i}\right), i=1,2$, and let $\widehat{\Omega} \doteq \Omega+J_{1}^{*} B_{1}+J_{2}^{*} B_{2}$. Then

(i) the maps $J_{i}:\left(V, L_{\widehat{\Omega}}\right) \longrightarrow\left(W_{i}, \tau_{B_{i}}\left(L_{\pi_{i}}\right)\right), i=1,2$, are Dirac;

(ii) the form $\widehat{\Omega}$ is symplectic if and only if $\tau_{B_{i}}\left(L_{\pi_{i}}\right), i=1,2$, are Poisson;

(iii) $\left(\operatorname{ker} J_{1}\right)^{\widehat{\Omega}}=\operatorname{ker} J_{2}+\operatorname{ker} \widehat{\Omega}$.

Proof: By Lemma 2.11, $J_{2}:\left(V, L_{\Omega+J_{2}^{*} B_{2}}\right) \longrightarrow\left(W_{2}, \tau_{B_{2}}\left(L_{\pi_{2}}\right)\right)$ is Dirac, and, by Lemma 2.14, $J_{1}:\left(V, L_{\Omega+J_{2}^{*} B_{2}}\right) \longrightarrow\left(W_{1}, \pi_{1}\right)$ is also Dirac. Again by Lemma 2.11, it follows that $J_{1}:\left(V, L_{\widehat{\Omega}}\right) \longrightarrow\left(W_{1}, \tau_{B_{1}}\left(L_{\pi_{1}}\right)\right)$ is Dirac. By Lemma $2.15,\left(\operatorname{ker} J_{2}\right)^{\Omega+J_{2}^{*} B_{2}}=\left(\operatorname{ker} J_{2}\right)^{\Omega}=\operatorname{ker} J_{1}$. So condition $(2.17)$ is satisfied, and Lemma 2.14 implies that $J_{2}:\left(V, L_{\widehat{\Omega}}\right) \longrightarrow\left(W_{2}, \tau_{B_{2}}\left(L_{\pi_{2}}\right)\right)$ is a Dirac map. This proves $i$ ).

In order to prove $i$ ), let us assume that $\widehat{\Omega}$ is symplectic. It follows from $i$ ) and Prop. 2.2(i) that $\tau_{B_{1}}\left(L_{\pi_{1}}\right)$ and $\tau_{B_{2}}\left(L_{\pi_{2}}\right)$ are Poisson. Conversely, if $\tau_{B_{1}}\left(L_{\pi_{1}}\right)$ is Poisson, then $\Omega+J_{1}^{*} B_{1}$ is symplectic, by Lemma 2.12. It follows, again from Lemma 2.12, that if $\tau_{B_{2}}\left(L_{\pi_{2}}\right)$ is also Poisson, then $\Omega+J_{1}^{*} B_{1}+J_{2}^{*} B_{2}$ is symplectic.

We now prove $i$ iii). Clearly, $\left(\operatorname{ker} J_{1}\right)^{\widehat{\Omega}}=\left(\operatorname{ker} J_{1}\right)^{\Omega+J_{2}^{*} B_{2}}=\operatorname{ker} J_{2}+\operatorname{ker}\left(\Omega+J_{2}^{*} B_{2}\right)$, by Lemma 2.15. Thus ker $J_{2}+\operatorname{ker} \widehat{\Omega} \subseteq\left(\operatorname{ker} J_{1}\right)^{\widehat{\Omega}}$. On the other hand, again by Lemma $2.15, \operatorname{ker}\left(\Omega+J_{2}^{*} B_{2}\right) \subseteq$ $\operatorname{ker} J_{1}$. So $\operatorname{ker} \widehat{\Omega} \cap \operatorname{ker} J_{1}=\operatorname{ker}\left(\Omega+J_{2}^{*} B_{2}\right)$, and therefore $\operatorname{ker}\left(\Omega+J_{2}^{*} B_{2}\right) \subseteq \operatorname{ker} \widehat{\Omega}$. Hence ker $J_{2}+\operatorname{ker}\left(\Omega+J_{2}^{*} B_{2}\right) \subseteq \operatorname{ker} J_{2}+\operatorname{ker} \widehat{\Omega}$, and the result follows.

\subsection{Dirac structures on manifolds}

Let $M$ be a smooth manifold. A Dirac structure on $M[10]$ is a subbundle $L \subset T M \oplus T^{*} M$ which determines linear Dirac structures pointwise and whose sections are closed under the Courant bracket [ , ] : $\Gamma\left(T M \oplus T^{*} M\right) \times \Gamma\left(T M \oplus T^{*} M\right) \longrightarrow \Gamma\left(T M \oplus T^{*} M\right)$,

$$
((X, \omega),(Y, \mu)) \mapsto\left([X, Y], L_{X} \mu-L_{Y} \omega+\frac{1}{2} d(\omega(Y)-\mu(X)) .\right.
$$

Let $\left(M, L_{M}\right)$ and $\left(N, L_{N}\right)$ be Dirac manifolds. A smooth map $\phi: M \longrightarrow N$ is a (forward) Dirac map if $\mathcal{F} T_{x} \phi\left(\left(L_{M}\right)_{x}\right)=\left(L_{N}\right)_{\phi(x)}$ for all $x \in M$.

Example 2.17 Let $\Omega \in \Omega^{2}(M)$ (resp. $\pi \in \chi^{2}(M)$ ). As discussed in Section 2.1, $L=$ $\operatorname{graph}(\Omega) \subset T M \oplus T^{*} M$ (resp. $L=\operatorname{graph}(\pi)$ ) defines a pointwise linear Dirac structure. In this case, the extra condition involving the Courant bracket in the definition of a Dirac 
manifold is equivalent to the integrability condition $d \Omega=0$ (resp. $[\pi, \pi]=0$, where [, ] is the Schouten bracket). Hence pre-symplectic and Poisson structures on $M$ are particular cases of Dirac structures.

The Courant bracket (2.18) does not satisfy the Jacobi identity in general. However, the Jacobi identity does hold when this bracket is restricted to sections of a Dirac subbundle $L \subset$ $T M \oplus T^{*} M$, and it defines a Lie algebroid structure on $L$ with anchor map $\left.\rho\right|_{L}$, where $\rho$ : $T M \oplus T^{*} M \longrightarrow T M$ is the natural projection. When $L=\operatorname{graph}(\pi)$, where $\pi$ is a Poisson structure, the Lie algebroid structure on $L$ is isomorphic to the natural Lie algebroid structure on $T^{*} M$ via the projection $\rho^{*}: T M \oplus T^{*} M \longrightarrow T^{*} M$.

A Dirac manifold $(M, L)$ carries a (singular) pre-symplectic foliation: the leaves are the orbits of the corresponding Lie algebroid and the leafwise pre-symplectic structure is defined as in Prop. 2.2(i). This foliation is symplectic if and only if $L=\operatorname{graph}(\pi)$ for a Poisson structure $\pi$.

As in the linear case, one can think of a Dirac structure $L$ on $M$ as a "Poisson structure on the leaf space $M / \mathcal{K}$ ", where $\mathcal{K}$ is the characteristic foliation of the leafwise pre-symplectic form $\Omega_{L}$. More precisely, if $(M, L)$ is a Dirac manifold, we define its set of admissible functions by

$$
\mathcal{A} \doteq\left\{f \in C^{\infty}(M)|d f|_{\operatorname{ker}\left(\Omega_{L}\right)}=0\right\}=\left\{f \in C^{\infty}(M) \mid d f \in \rho^{*}(L)\right\} .
$$

If $f \in \mathcal{A}$, there exists $X \in \chi(M)$ such that $(X, d f) \in L$; we call $X$ a hamiltonian vector field of $f$ and denote it by $X_{f}$. Note that hamiltonian vector fields of admissible functions are defined up to vector fields in $\operatorname{ker} \Omega_{L}$. If $f, g \in \mathcal{A}$, then the bracket

$$
\{f, g\} \doteq \Omega_{L}\left(X_{f}, X_{g}\right)
$$

is well defined and makes $\mathcal{A}$ into a Poisson algebra. Clearly, if $L$ comes from a Poisson structure, then $\mathcal{A}$ is just the Poisson algebra $\left(C^{\infty}(M),\{\},\right)$. If the characteristic foliation $\mathcal{K}$ of $\Omega_{L}$ is simple, i.e., if $M / \mathcal{K}$ is a smooth manifold and pr : $M \longrightarrow M / \mathcal{K}$ is a submersion, then $\mathcal{A} \cong C^{\infty}(M / \mathcal{K})$ and there is a naturally defined Poisson structure $\pi_{L}$ on $M / \mathcal{K}$ in such a way that pr is a Dirac map (Example 2.9).

Gauge transformations of Dirac structures are defined analogously to the linear case: if $L$ is a Dirac structure on $M$ and $B$ a closed 2-form, we set

$$
\tau_{B}(L)=\{(X, \eta+B(X)),(X, \eta) \in L\} .
$$

The closedness of $B$ guarantees that $\tau_{B}(L)$ satisfies the integrability condition with respect to the Courant bracket. As in the linear case, two Dirac structures on $M$ in the same $\tau$-orbit are called gauge equivalent.

As observed in [27, Sec. 3], for a Poisson structure $\pi$ on $M, \tau_{B}(\pi)$ is Poisson if and only if the endomorphism $1+B \pi: T^{*} M \longrightarrow T^{*} M$ is invertible. In this case,

$$
\tau_{B}(\pi)=\pi(1+B \pi)^{-1}
$$

Gauge-equivalent Dirac structures share many properties: for instance, they have the same leaf decomposition (though the pre-symplectic forms on the leaves differ by the pullbacks of $B$ (see $(2.15))$ ) and their corresponding Lie algebroids are isomorphic [27]. In particular, gaugeequivalent Poisson structures have isomorphic Poisson cohomology. 


\section{Pre-dual pairs and reduction}

A dual pair [29] consists of a symplectic manifold $(S, \Omega)$, Poisson manifolds $\left(M_{1}, \pi_{1}\right),\left(M_{2}, \pi_{2}\right)$, and Poisson maps $J_{i}: S \longrightarrow M_{i}, i=1,2$ with symplectically orthogonal fibers, i.e.,

$$
\operatorname{ker} T_{x} J_{1}=\left(\operatorname{ker} T_{x} J_{2}\right)^{\Omega} \text {. }
$$

A dual pair is called full if the maps $J_{1}, J_{2}$ are surjective submersions, and complete if these Poisson maps are complete (see e.g. [6]).

If $M_{1} \stackrel{J_{1}}{\leftarrow} S \stackrel{J_{2}}{\rightarrow} M_{2}$ is a full dual pair, then

$$
\begin{gathered}
\operatorname{ker} T_{x} J_{1}=\left\{X_{J_{2}^{*} f}, f \in C^{\infty}\left(M_{2}\right)\right\}, \text { and } \\
\left\{J_{1}^{*}\left(C^{\infty}\left(M_{1}\right)\right), J_{2}^{*}\left(C^{\infty}\left(M_{2}\right)\right)\right\}=0 .
\end{gathered}
$$

In order to deal with Dirac structures, we generalize the notion of a dual pair as follows.

Definition 3.1 A pre-dual pair is a pre-symplectic manifold $(S, \Omega)$, Dirac manifolds $\left(M_{1}, L_{1}\right)$, $\left(M_{2}, L_{2}\right)$ and Dirac maps $J_{i}: S \longrightarrow M_{i}, i=1,2$, such that

$$
\left(\operatorname{ker} T_{x} J_{1}\right)^{\Omega}=\operatorname{ker} T_{x} J_{2}+\operatorname{ker} \Omega \text { and }\left(\operatorname{ker} T_{x} J_{2}\right)^{\Omega}=\operatorname{ker} T_{x} J_{1}+\operatorname{ker} \Omega .
$$

As in the case of dual pairs, we represent pre-dual pairs by a diagram

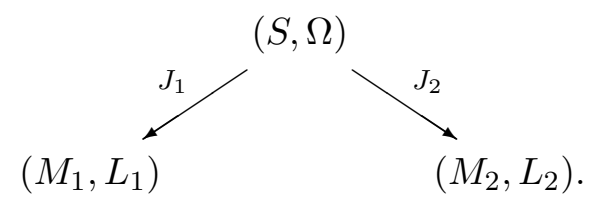

As before, a pre-dual pair will be called full if each $J_{i}$ is a surjective submersion.

Proposition 3.2 Consider a pre-dual pair $M_{1} \stackrel{J_{1}}{\leftarrow} S \stackrel{J_{2}}{\rightarrow} M_{2}$. If $S$ is symplectic, then $M_{1}$ and $M_{2}$ are automatically Poisson, and we have a dual pair in the usual sense.

Proof: If $\operatorname{ker}(\Omega)=0$, then $\operatorname{ker}\left(\Omega_{L_{i}}\right)=T J_{i}(\operatorname{ker} \Omega)=0$, and hence $L_{i}$ is automatically Poisson. The maps $J_{i}, i=1,2$, are Dirac, and hence Poisson, and the orthogonality property (3.4) reduces to $(3.1)$.

We now fix a full pre-dual pair $M_{1} \stackrel{J_{1}}{\leftarrow} S \stackrel{J_{2}}{\rightarrow} M_{2}$.

Proposition 3.3 We have the following generalization of (3.2):

$$
\operatorname{ker} T_{x} J_{1}+\operatorname{ker} \Omega=\left(\operatorname{ker} T_{x} J_{2}\right)^{\Omega}=\left\{X_{J_{2}^{*} f} \mid f \in C^{\infty}\left(M_{2}\right)\right\} .
$$

Proof: Clearly, if $v \in \operatorname{ker} T_{x} J_{2}, \Omega\left(X_{J_{2}^{*} f}, v\right)=d_{x}\left(J_{2}^{*} f\right)(v)=d f\left(T_{x} J_{2}(v)\right)=0$. On the other hand, if $u \in\left(\operatorname{ker} T_{x} J_{2}\right)^{\Omega}, \eta=\Omega_{x}(u) \in T_{x}^{*} S$ vanishes along $\operatorname{ker} T_{x} J_{2}$. Since $J_{2}$ is a submersion, in a neighborhood of $x$ we can choose an exact 1-form $d g$ with $d_{x} g=\eta$ and such that $d g$ vanishes on the distribution ker $T J_{2}$. Hence $g$ is constant along the $J_{2}$-fibers and can be written in the form $J_{2}^{*} f$ for $f \in C^{\infty}\left(M_{2}\right)$. Around $x, u=X_{J_{2}^{*} f}$.

The next result generalizes property (3.3). 
Proposition 3.4 Let $\mathcal{A}$ be the algebra of admissible functions on $S$, and let $\mathcal{A}_{i}$ be the algebra of admissible functions on $M_{i}$. Then

i) $J_{i}^{*} \mathcal{A}_{i} \subseteq \mathcal{A}$,

ii) $\left\{J_{1}^{*} \mathcal{A}_{1}, J_{2}^{*} \mathcal{A}_{2}\right\}=0$,

iii) $\left\{J_{i}^{*} f, J_{i}^{*} g\right\}=J_{i}^{*}\{f, g\}$, for $f, g \in \mathcal{A}_{i}$.

Proof: By Prop. 2.6, $T J_{i}(X) \in \operatorname{ker} \Omega_{L_{i}}$. So, if $X \in \operatorname{ker} \Omega$ and $f \in \mathcal{A}_{i}$, then $d\left(J_{i}^{*} f\right)(X)=$ $d f\left(T J_{i}(X)\right)=0$, and $\left.i\right)$ is proven.

For $i i)$, note that if $f_{i} \in \mathcal{A}_{i}$, then $\left\{J_{1}^{*} f_{1}, J_{2}^{*} f_{2}\right\}=\Omega\left(X_{J_{1}^{*} f_{1}}, X_{J_{2}^{*} f_{2}}\right)=0$ by Prop. 3.4.

Finally, since $J_{i}$ is a Dirac map, $\left(X, J_{i}^{*} d g\right) \in L$ implies that $\left(T_{x} J_{i}(X), d g\right) \in \mathcal{F} T_{x} J_{i}(L)=L_{i}$. This means that $T_{x} J_{i}\left(X_{J_{i}^{*} g}\right)=X_{g}$. Now, if $f, g \in \mathcal{A}_{i}$, then

$$
\left\{J_{i}^{*} f, J_{i}^{*} g\right\}(x)=d_{x}\left(J_{i}^{*} f\right)\left(X_{J_{i}^{*} g}\right)=d_{J_{i}(x)} f\left(T_{x} J_{i}\left(X_{J_{i}^{*} g}\right)\right)=d_{J_{i}(x)} f\left(X_{g}\right)=\{f, g\}\left(J_{i}(x)\right),
$$

and the result follows.

Suppose that the characteristic foliations of $\Omega$ and $\Omega_{L_{i}}, \mathcal{K}$ and $\mathcal{K}_{i}$, respectively, are simple. Recall that $S / \mathcal{K}$ has an induced symplectic structure $\Omega_{\text {red }}$, while $M_{i} / \mathcal{K}_{i}, i=1,2$, carry induced Poisson structures $\pi_{i}$ so that the projections pr $: S \longrightarrow S / \mathcal{K}$ and $\operatorname{pr}_{i}: S \longrightarrow M_{i} / \mathcal{K}_{i}$ are Dirac maps.

Theorem 3.5 Let $M_{1} \stackrel{J_{1}}{\leftarrow} S \stackrel{J_{2}}{\rightarrow} M_{2}$ be a full pre-dual pair. The maps $J_{i}: S \longrightarrow M_{i}$ induce surjective submersions on the quotient, $j_{i}: S / \mathcal{K} \longrightarrow M_{i} / \mathcal{K}_{i}$, in such a way that

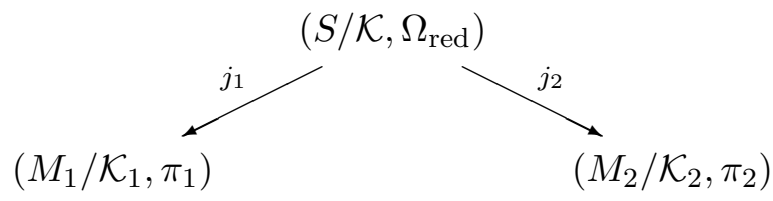

is a full dual pair.

Proof: By Prop. 2.6(i), $T J_{i}(\operatorname{ker} \Omega)=\operatorname{ker} \Omega_{L_{i}}$. Hence if $x$ and $y$ belong to the same leaf of $\mathcal{K}$, then $J_{i}(x)$ and $J_{i}(y)$ belong to the same leaf of $\mathcal{K}_{i}$. So the map $j_{i}: S / \mathcal{K} \longrightarrow M_{i} / \mathcal{K}_{i}, j_{i}(\operatorname{pr}(x))=$ $\operatorname{pr}_{i}\left(J_{i}(x)\right)$ is well defined and is a surjective submersion. It follows from Lemma 2.10 that $j_{i}$ is a Poisson map. Finally, a simple computation shows that $\left(\operatorname{ker} T j_{i}\right)^{\Omega_{\mathrm{red}}}=\operatorname{pr}\left(\left(\operatorname{ker} T J_{i}\right)^{\Omega}\right)$. Hence (3.4) implies that $\left(\operatorname{ker} T j_{1}\right)^{\Omega_{\text {red }}}=\operatorname{ker} T j_{2}$.

We obtain examples of pre-dual pairs from gauge transformations of Poisson structures in dual pairs as follows.

Theorem 3.6 Let $\left(M_{1}, \pi_{1}\right) \stackrel{J_{1}}{\leftarrow}(S, \Omega) \stackrel{J_{2}}{\rightarrow}\left(M_{2}, \pi_{2}\right)$ be a full dual pair, and let $B_{i}$ be a closed 2 -form on $M_{i}, i=1,2$. Let $\widehat{\Omega}=\Omega+J_{1}^{*} B_{1}+J_{2}^{*} B_{2}$. Then

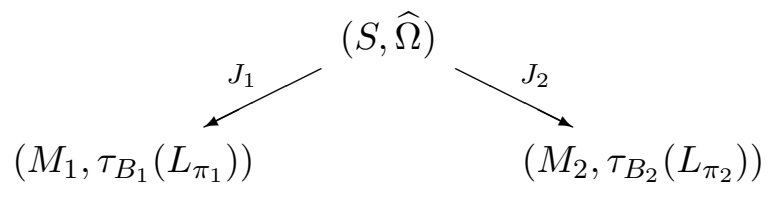


is a full pre-dual pair. Moreover, $\widehat{\Omega}$ is symplectic if and only if $\tau_{B_{i}}\left(L_{\pi_{2}}\right), i=1,2$, are Poisson, in which case they form a dual pair.

Proof: The result is a consequence of Theorem 2.16.

\section{Gauge equivalence of symplectic groupoids}

In this section we will apply the results of Section 3 to dual pairs coming from symplectic groupoids.

A symplectic groupoid [30] is a symplectic manifold $(G, \Omega)$ which is a Lie groupoid such that the graph $\gamma_{m}=\left\{(x, y, m(x, y)),(x, y) \in G_{2}\right\}$ of the multiplication is lagrangian in $G \times G \times \bar{G}$ (as usual, $G_{2}$ denotes the set of composable pairs). We denote the source (resp. target) map of $G$ by $\alpha$ (resp. $\beta$ ), the identity embedding by $\epsilon: G_{0} \hookrightarrow G$, and the inversion by $i: G \longrightarrow G$. We recall that there exists a unique Poisson structure $\pi$ on $G_{0}$ making $\alpha$ (resp. $\beta$ ) into a Poisson (resp. anti-Poisson) map.

Let $(M, \pi)$ be an integrable Poisson manifold, with symplectic groupoid $(G, \Omega, \alpha, \beta)$. Since Lie algebroids corresponding to gauge-equivalent Dirac structures are isomorphic, all the Lie algebroids of Dirac structures in the $\tau$-orbit of $\pi$ can be integrated to a Lie groupoid isomorphic to $(G, \alpha, \beta)$. We now discuss the effect of a gauge transformation $\tau_{B}$ on the symplectic form $\Omega$.

Let $B$ be a closed 2-form on $M$, and consider the 2-form $\Omega_{B} \doteq \Omega+\alpha^{*} B-\beta^{*} B$ on $G$.

Theorem 4.1 If $\pi_{B} \doteq \tau_{B}(\pi)$ is Poisson, then $G_{B} \doteq\left(G, \Omega_{B}, \alpha, \beta\right)$ is a symplectic groupoid integrating $\left(M, \pi_{B}\right)$.

Proof: By Theorem 2.16, $\Omega_{B}$ is symplectic. We must check that the graph

$$
\gamma_{m}=\left\{(x, y, m(x, y)),(x, y) \in G_{2}\right\}
$$

is lagrangian in $G_{B} \times G_{B} \times \overline{G_{B}}$. Let $(x, y) \in G_{2}$, and consider a curve $(x(t), y(t))$ in $G_{2}$ with $(x(0), y(0))=(x, y)$. Let $(u, v)=\left(x^{\prime}(0), y^{\prime}(0)\right)$. Then $\left(u, v, T_{(x, y)} m(u, v)\right) \in T_{p} \gamma_{m}, p=$ $(x, y, m(x, y))$ and any element in $T_{p} \gamma_{m}$ is of this form.

Differentiating the identities $\alpha(m(x, y))=\alpha(x), \beta(m(x, y))=\beta(y)$ and $\beta(x)=\alpha(y)$, we get

$$
\begin{gathered}
\operatorname{T\alpha Tm}(u, v)=T \alpha(u), \quad T \beta T m(u, v)=T \beta(v), \\
T \beta(u)=T \alpha(v) .
\end{gathered}
$$

Therefore, if $\left(u_{1}, v_{1}, \operatorname{Tm}\left(u_{1}, v_{1}\right)\right),\left(u_{2}, v_{2}, \operatorname{Tm}\left(u_{2}, v_{2}\right)\right) \in T_{p} \gamma_{m}$, we have

$$
\begin{aligned}
& \left(\Omega_{B} \times \Omega_{B} \times\left(-\Omega_{B}\right)\right)\left(\left(u_{1}, v_{1}, T m\left(u_{1}, v_{1}\right),\left(u_{2}, v_{2}, T m\left(u_{2}, v_{2}\right)\right)\right)=\right. \\
& B\left(T \alpha\left(u_{1}\right), T \alpha\left(u_{2}\right)\right)-B\left(T \beta\left(u_{1}\right), T \beta\left(u_{2}\right)\right)+B\left(T \alpha\left(v_{1}\right), T \alpha\left(v_{2}\right)\right)-B\left(T \beta\left(v_{1}\right), T \beta\left(v_{2}\right)\right)- \\
& B\left(T \alpha T m\left(u_{1}, v_{1}\right), T \alpha T m\left(u_{2}, v_{2}\right)\right)+B\left(T \beta T m\left(u_{1}, v_{1}\right), T \beta T m\left(u_{2}, v_{2}\right)\right)=0,
\end{aligned}
$$

Hence $\gamma_{m}$ is lagrangian in $G_{B} \times G_{B} \times \overline{G_{B}}$ and $G_{B}$ is a symplectic groupoid.

By Theorem 3.6, $\alpha:\left(G, \Omega_{B}\right) \longrightarrow\left(M, \pi_{B}\right)$ is a Poisson map. Since there is a unique Poisson structure on the identity section of a symplectic groupoid with this property, the Poisson structure induced by $\Omega_{B}$ on $M$ is $\pi_{B}$. 
Thus, the effect of applying a gauge transformation $\tau_{B}$ to the Poisson structure of the identity section of a symplectic groupoid is the following change of the symplectic form on the groupoid:

$$
\Omega \stackrel{\tau_{B}}{\longmapsto} \Omega_{B}=\Omega+\alpha^{*} B-\beta^{*} B .
$$

Note that, in general, $\tau_{B}(\pi)$ is not Poisson, and the form $\Omega_{B}$ on $G$ is degenerate. So

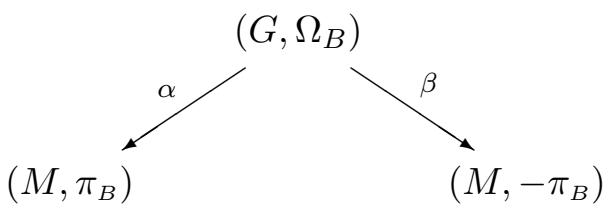

is generally just a pre-dual pair. In this context, one is naturally led to consider groupoids equipped with pre-symplectic forms; the associated integration problem is whether a Dirac structure with an integrable Lie algebroid can be integrated to such a "pre-symplectic groupoid" (we believe an analog of the constructions in [7,13] should clarify this question). Similarly, considering gauge transformations on Poisson groupoids [31], we are led to the more general notion of "Dirac groupoids". The development of these ideas is the subject of work in progress.

\section{Morita equivalence of gauge-equivalent Poisson structures}

In this section, we compare the notions of gauge and Morita equivalence for integrable Poisson manifolds.

Two Poisson manifolds $\left(M_{1}, \pi_{1}\right),\left(M_{2}, \pi_{2}\right)$ are called Morita equivalent [33] if there exists a symplectic manifold $(S, \Omega)$ and Poisson maps $J_{i}: S \longrightarrow M_{i}, i=1,2$, so that

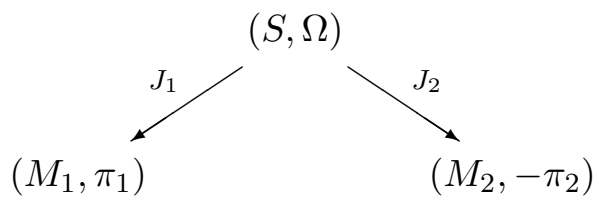

is a complete full dual pair with $J_{i}$-connected and $J_{i}$-simply-connected fibers. In this case we call this diagram a Morita equivalence bimodule.

Let $(M, \pi)$ be an integrable Poisson manifold with $\alpha$-connected and $\alpha$-simply-connected symplectic groupoid $(G, \Omega, \alpha, \beta)$. Let $B$ be a closed 2-form on $M$ such that $\pi_{B}=\tau_{B}(\pi)$ is Poisson.

Theorem 5.1 The Poisson manifolds $(M, \pi),\left(M, \pi_{B}\right)$ are Morita equivalent, with Morita equivalence bimodule $(G, \widehat{\Omega}, \alpha, \beta)$, where $\widehat{\Omega}=\Omega-\beta^{*} B$.

Proof: By Theorem 3.6, $(M, \pi) \stackrel{\alpha}{\leftarrow}(G, \widehat{\Omega}) \stackrel{\beta}{\rightarrow}\left(M, \tau_{-B}(-\pi)\right)$ is a full dual pair with connected and simply connected fibers. Since $\left.\tau_{-B}(-\pi)\right)=-\tau_{B}(\pi), \beta:(G, \widehat{\Omega}) \longrightarrow\left(M, \tau_{B}(\pi)\right)$ is antiPoisson, and it only remains to show that this dual pair is complete.

Let $X_{h}$ denote the hamiltonian vector field of $h$ with respect to $\Omega$. Let $\widehat{X}_{h}$ and $X_{h}^{B}$ denote the hamiltonian vector fields with respect to $\widehat{\Omega}$ and $\Omega_{B}=\Omega+\alpha^{*} B-\beta^{*} B$.

Claim. We have the following relations between the hamiltonian vector fields:

$$
\widehat{X}_{\alpha^{*} f}=X_{\alpha^{*} f} \text { and } \quad \widehat{X}_{\beta^{*} f}=X_{\beta^{*} f}^{B}, \text { for all } f \in C^{\infty}(M)
$$


Proof: Since $X_{\alpha^{*} f} \in \operatorname{ker} \beta, \beta^{*} B\left(X_{\alpha^{*} f}\right)=0$. Hence $\widehat{\Omega}\left(X_{\alpha^{*} f}\right)=\Omega\left(X_{\alpha^{*} f}\right)=d\left(\alpha^{*} f\right)$. Therefore, $\widehat{X}_{\alpha^{*} f}=X_{\alpha^{*} f}$. The other relation can be derived analogously.

Let now $f \in C^{\infty}(M)$ be a complete function with respect to $\pi$ (resp. $\pi_{B}$ ). We must check that $\widehat{X}_{\alpha^{*} f}$ (resp. $\widehat{X}_{\beta^{*} f}$ ) is complete.

By our claim, $\widehat{X}_{\alpha^{*} f}=X_{\alpha^{*} f}$, which is complete, since $\alpha:(G, \Omega) \longrightarrow(M, \pi)$ is the target map of a symplectic groupoid [9, Chp. III]. Analogously, since $\left(G, \Omega_{B}, \alpha, \beta\right)$ is a symplectic groupoid for $\left(M, \pi_{B}\right)$, it follows that $\beta:\left(G, \Omega_{B}\right) \longrightarrow\left(M, \pi_{B}\right)$ is complete, and hence $\widehat{X}_{\beta^{*} f}=X_{\beta^{*} f}^{B}$ is complete as well.

It is clear that Morita equivalent Poisson structures on a manifold $M$ need not be gauge equivalent, as their leaf decompositions are not necessarily the same. More generally, we say that two Poisson structures $\pi$ and $\pi^{\prime}$ on $M$ are gauge equivalent up to Poisson diffeomorphism if there exists a diffeomorphism $f: M \longrightarrow M$ such that $f_{*} \pi$ and $\pi^{\prime}$ are gauge equivalent. It is clear that integrable Poisson structures which are gauge equivalent up to Poisson diffeomorphism are still Morita equivalent. However, as the next example shows, one can have Morita equivalent Poisson structures on a manifold $M$ which are not gauge equivalent up to Poisson diffeomorphism.

Example 5.2 The example is based on non-cancellation properties of product manifolds (see [19] and references therein). Let $F_{1}, F_{2}$ and $B$ be closed smooth manifolds so that $F_{1}$ and $F_{2}$ have different homotopy types and $F_{1} \times B$ is diffeomorphic to $F_{2} \times B$ (see [8] for original examples). As discussed in [19], one can take $B=S^{q}$ and $F_{i}$ to be $S^{q}$-bundles over $S^{n}$, for $q$ and $n$ suitably chosen (and large). Hence we can assume that $B$ and $F_{i}$ are simply connected.

Let $E$ be the total space of these trivial fibrations, with diffeomorphisms $E \stackrel{\varphi_{i}}{\longrightarrow} B \times F_{i}, i=$ 1,2 . Let $M=T^{*} E$. If $\varphi_{i}^{\sharp}$ denotes the natural cotangent lift of $\varphi_{i}$, we obtain the diffeomorphisms

$$
\varphi_{i}^{\sharp}: M \longrightarrow T^{*} B \times T^{*} F_{i}, \quad i=1,2 .
$$

Since the transformations in the structure group of the fiber bundle

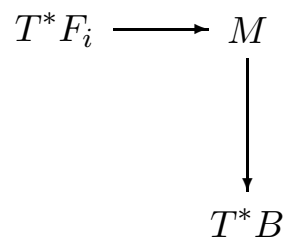

are cotangent lifts of diffeomorphisms of $F_{i}$, they preserve the canonical symplectic forms on $T^{*} F_{i}, i=1,2$. Thus $\varphi_{i}^{\sharp}$ makes $M$ into a bundle of symplectic manifolds in the sense of [18], defining a Poisson structure $\pi_{i}$ on $M$. As $F_{1}$ and $F_{2}$ have different homotopy types, so do $T^{*} F_{1}$ and $T^{*} F_{2}$, and hence $\pi_{1}$ and $\pi_{2}$ cannot be gauge equivalent up to Poisson diffeomorphism.

Finally note that since $F_{1}, F_{2}$ and $B$ are simply connected, so are $T^{*} F_{1}, T^{*} F_{2}$ and $T^{*} B$, and [18, Thm. 3] implies that the symplectic structure along the fibers of $T^{*} F_{i} \rightarrow M \rightarrow T^{*} B$ admits a closed extension. Hence the fundamental class [14] of $\left(M, \pi_{i}\right)$ vanishes, and [33, Thm. 4.3] implies that $\left(M, \pi_{i}\right)$ is Morita equivalent to the base $T^{*} B$ equipped with the zero Poisson structure. By transitivity, $\left(M, \pi_{1}\right)$ and $\left(M, \pi_{2}\right)$ are Morita equivalent. 


\section{Gauge and Morita equivalence of topologically stable Poisson structures on surfaces}

Let $\Sigma$ be a compact connected oriented surface. Since for dimensional reasons any bivector field on $\Sigma$ is Poisson, Poisson structures on $\Sigma$ form a vector space.

For $n \geq 0$, let $\mathscr{G}_{n}(\Sigma)$ be the set of Poisson structures $\pi$ on $\Sigma$ such that

- the zero set $\{p \in \Sigma \mid \pi(p)=0\}$ consists of $n$ smooth disjoint curves $\gamma_{1}(\pi), \cdots, \gamma_{n}(\pi)$;

- $\pi$ vanishes linearly on each of the curves $\gamma_{1}(\pi), \cdots, \gamma_{n}(\pi)$.

Let $\mathscr{G}(\Sigma) \doteq \bigsqcup_{n \geq 0} \mathscr{G}_{n}(\Sigma)$. For $n \geq 1$ the symplectic leaves of $\pi \in \mathscr{G}(\Sigma)$ are the points in the zero set $\bigsqcup_{i=1}^{n} \gamma_{i}$ and the connected components of $\Sigma \backslash \bigsqcup_{i=1}^{n} \gamma_{i}$; for $n=0$ the structure is symplectic. We call the Poisson structures in $\mathscr{G}(\Sigma)$ topologically stable, since the topology of their zero sets is preserved under small perturbations.

Choosing a non-degenerate Poisson structure $\pi_{0}$ on $\Sigma$, we can identify the space of Poisson structures on $\Sigma$ with $C^{\infty}(\Sigma)$ : any $\pi$ is represented in the form $\pi=f \cdot \pi_{0}$ for $f \in C^{\infty}(\Sigma)$. Under this identification, $\mathscr{G}(\Sigma)$ corresponds to the space of smooth functions for which 0 is a regular value. This implies that the set of topologically stable Poisson structures $\mathscr{G}(\Sigma)$ is generic, i.e., $\mathscr{G}(\Sigma)$ is an open dense subset of the space of all Poisson structures on $\Sigma$ endowed with the Whitney $C^{\infty}$ topology.

As shown in [25], the classification of Poisson structures in $\mathscr{G}(\Sigma)$ up to Poisson isomorphisms depends on a finite number of invariants. In order to recall what these invariants are, we need a few definitions.

For a Poisson manifold $(M, \pi)$, let $\nu$ be a volume form on $M$. The modular vector field $X^{\nu}$ of $\pi$ with respect to $\nu[32]$ is defined by the formula

$$
X^{\nu} h \doteq \frac{L_{X_{h}} \nu}{\nu}, \quad h \in C^{\infty}(M) .
$$

This vector field measures the degree of invariance of $\nu$ under the flows of hamiltonian vector fields; in particular, $X^{\nu}=0$ if and only if $L_{X_{h}} \nu=0$ for all $h \in C^{\infty}(M)$. The modular vector field $X^{\nu}$ is Poisson (i.e., its flow preserves $\pi$ ), and, if $\nu^{\prime}$ is a different volume form, one has

$$
X^{\nu^{\prime}}=X^{\nu}+X_{-\log k}
$$

where $k$ is the nowhere zero ratio $k=\left|\frac{\nu^{\prime}}{\nu}\right|$. Thus the class of $X^{\nu}$ in the first Poisson cohomology $H_{\pi}^{1}(M)$ (i.e., its equivalence class modulo hamiltonian vector fields) is independent of $\nu$. This defines an element in $H_{\pi}^{1}(M)$ called the modular class and denoted by $\mu_{(M, \pi)}$.

Suppose that the Poisson tensor $\pi$ on $M$ vanishes on a closed curve $\gamma \subset M$, and is nonzero away from $\gamma$ in a neighborhood of $\gamma$. Since the modular vector field $X^{\nu}$ preserves the Poisson structure, its flow must take the zero set of $\pi$ to the zero set of $\pi$. Thus the flow of $X^{\nu}$ takes $\gamma$ to $\gamma$ and so $X^{\nu}$ must be tangent to $\gamma$. Moreover, for another choice of volume form $\nu^{\prime}$, we have

$$
\left.X^{\nu^{\prime}}\right|_{\gamma}=\left.X^{\nu}\right|_{\gamma}+\left.\left(X_{-\log \left|\frac{\nu^{\prime}}{\nu}\right|}\right)\right|_{\gamma}=\left.X^{\nu}\right|_{\gamma}
$$

since all hamiltonian vector fields are zero when restricted to the zero curve $\gamma$. It follows that the restriction of the modular vector field $X^{\nu}$ to $\gamma$ is independent of $\nu$, and hence induces an orientation on $\gamma$. As was observed in [26], the period of the flow of this vector field around $\gamma$ 
is an invariant of the Poisson structure $\pi$. We denote this number by $T_{\gamma}(M, \pi)$ (or, for short, $T_{\gamma}(\pi)$ when it is clear what $M$ is).

For $\pi \in \mathscr{G}_{n}(\Sigma)$, let $Z(\pi)$ denote its zero set, consisting on $n$ disjoint curves, taken with the induced orientations. The main result of [25] states that Poisson structures in $\mathscr{G}_{n}(\Sigma)$ are completely classified up to (orientation-preserving) Poisson isomorphisms by the class of $Z(\pi)$ modulo orientation-preserving diffeomorphisms of $\Sigma$, the $n$ modular periods of $\pi$ around each connected component of $Z(\pi)$ and the regularized Liouville volume $V(\pi)$ (which is a certain regularized sum of symplectic volumes of two-dimensional leaves, taken with appropriate signs).

We shall now consider the questions of gauge and Morita equivalence of Poisson structures in $\mathscr{G}(\Sigma)$.

\subsection{Gauge equivalence in $\mathscr{G}(\Sigma)$}

The obvious necessary condition for two Poisson structures $\pi, \pi^{\prime} \in \mathscr{G}(\Sigma)$ to be gauge equivalent is $Z(\pi)=Z\left(\pi^{\prime}\right)$, i.e. the zero sets of both structures, with the induced orientations, should be the same.

Proposition 6.1 Let $\pi, \pi^{\prime} \in \mathscr{G}_{n}(\Sigma)$ be Poisson structures with $Z(\pi)=Z\left(\pi^{\prime}\right)=\bigsqcup_{i=1}^{n} \gamma_{i}$. If they are gauge equivalent, then their modular periods are the same around all the zero curves, i.e. $T_{\gamma_{i}}(\pi)=T_{\gamma_{i}}\left(\pi^{\prime}\right)$, for $i=1, \ldots, n$.

Proof: Let $\pi=f \cdot \pi_{0}, \pi^{\prime}=f^{\prime} \cdot \pi_{0}$, where $f, f^{\prime} \in C^{\infty}(\Sigma)$ are functions vanishing linearly on $\gamma_{1}, \cdots, \gamma_{n}$ and non-zero elsewhere, and $\pi_{0}$ is a fixed nondegenerate Poisson structure on $\Sigma$.

For each $i=1, \ldots, n$, let $U_{i}=\left\{\left(z_{i}, \theta_{i}\right)|| z_{i} \mid<R_{i}, \theta_{i} \in[0,2 \pi]\right\}$ be a small annular neighborhood of the zero curve $\gamma_{i} \in Z(\pi)$ such that $Z(\pi) \cap U_{i}=\gamma_{i}$ and $\left.\pi\right|_{U_{i}}=f\left(z_{i}, \theta_{i}\right) \partial_{z_{i}} \wedge \partial_{\theta_{i}},\left.\pi^{\prime}\right|_{U_{i}}=$ $f^{\prime}\left(z_{i}, \theta_{i}\right) \partial_{z_{i}} \wedge \partial_{\theta_{i}}$. A simple computation shows that the modular vector fields along $\gamma_{i}$ are

$$
X_{\pi}=\frac{\partial f}{\partial z_{i}}\left(0, \theta_{i}\right) \partial_{\theta_{i}}, \quad X_{\pi^{\prime}}=\frac{\partial f^{\prime}}{\partial z_{i}}\left(0, \theta_{i}\right) \partial_{\theta_{i}}
$$

Suppose that $\pi^{\prime}=\tau_{B}(\pi)$ for a closed 2-form $B \in \Omega^{2}(\Sigma)$. Writing $B=b\left(z_{i}, \theta_{i}\right) d z_{i} \wedge d \theta_{i}$, it follows that

$$
f^{\prime}\left(z_{i}, \theta_{i}\right)=\frac{f\left(z_{i}, \theta_{i}\right)}{1+f\left(z_{i}, \theta_{i}\right) b\left(z_{i}, \theta_{i}\right)}
$$

which implies that

$$
\frac{\partial f}{\partial z_{i}}\left(0, \theta_{i}\right)=\frac{\partial f^{\prime}}{\partial z_{i}}\left(0, \theta_{i}\right)
$$

So $\pi$ and $\pi^{\prime}$ have equal modular vector fields along $\gamma_{i}$, and hence equal modular periods.

We remark that if $\pi \in \mathscr{G}_{n}(\Sigma)$ and $B$ is a closed 2-form on $\Sigma$ such that $\tau_{B}(\pi)$ is Poisson, then the regularized Liouville volumes of $\pi$ and $\tau_{B}(\pi)$ are related by $V\left(\tau_{B}(\pi)\right)=V(\pi)+\operatorname{Vol}(B)$, where $\operatorname{Vol}(B)=\int_{\Sigma} B$ is the Liouville volume of $B$.

We now discuss the converse of Proposition 6.1.

Theorem 6.2 Let $\pi, \pi^{\prime} \in \mathscr{G}_{n}(\Sigma)$ be two Poisson structures with $Z(\pi)=Z\left(\pi^{\prime}\right)=\bigsqcup_{i=1}^{n} \gamma_{i}$. If $T_{\gamma_{i}}(\pi)=T_{\gamma_{i}}\left(\pi^{\prime}\right)$, for $i=1, \ldots, n$, then $\pi$ and $\pi^{\prime}$ are gauge equivalent up to Poisson diffeomorphism. 
Proof: Let $\pi=f \cdot \pi_{0}, \pi^{\prime}=f^{\prime} \cdot \pi_{0}$, with $f$ and $f^{\prime}$ as in the proof of Prop. 6.1. By replacing, if necessary, $\pi^{\prime}$ by a Poisson diffeomorphic structure (with same zero set), we can assume that, for each $i=1, \ldots, n$, there is a small annular neighborhood $U_{i}=\left\{\left(z_{i}, \theta_{i}\right)|| z_{i} \mid<R_{i}, \theta_{i} \in[0,2 \pi]\right\}$ of $\gamma_{i} \in Z(\pi)$ such that $Z(\pi) \cap U_{i}=\gamma_{i}$ and $\left.\pi\right|_{U_{i}}=f\left(z_{i}\right) \partial_{z_{i}} \wedge \partial_{\theta_{i}},\left.\pi^{\prime}\right|_{U_{i}}=f^{\prime}\left(z_{i}\right) \partial_{z_{i}} \wedge \partial_{\theta_{i}}$ with $\left.f\right|_{U_{i}}=c_{i} z_{i}+O\left(z^{2}\right)$, and $\left.f^{\prime}\right|_{U_{i}}=c_{i}^{\prime} z_{i}+O\left(z^{2}\right)$. A simple computation (see (??)) shows that $c_{i}=\frac{2 \pi}{T_{\gamma_{i}}(\pi)}$ and $c_{i}^{\prime}=\frac{2 \pi}{T_{\gamma_{i}}\left(\pi^{\prime}\right)}$, so $c_{i}=c_{i}^{\prime}$.

Let $\omega=f^{-1} \omega_{0}$ (resp. $\omega^{\prime}=f^{\prime-1} \omega_{0}$ ) be the symplectic form corresponding to $\pi$ (resp. $\pi^{\prime}$ ) on $\Sigma \backslash Z(\pi)$, and define the 2 -form $B$ on $\Sigma \backslash Z(\pi)$ by

$$
B=\omega^{\prime}-\omega=\left(\frac{1}{f^{\prime}}-\frac{1}{f}\right) \omega_{0}
$$

It is simple to check that, since $c_{i}=c_{i}^{\prime}$, the function $\frac{1}{f^{\prime}}-\frac{1}{f}=\frac{f-f^{\prime}}{f f^{\prime}}$ extends to a smooth function on $\Sigma$. Hence the 2 -form $B$ can be extended to a (closed) 2-form on $\Sigma$, also denoted by $B$, with the property that $\pi^{\prime}=\tau_{B}(\pi)$.

For $\pi \in \mathscr{G}_{n}(\Sigma)$, the anchor $\tilde{\pi}: T^{*} \Sigma \rightarrow T \Sigma$ of the corresponding Lie algebroid is injective on the open dense set $\Sigma \backslash Z(\pi)$. According to [15, Thm. 1], a Lie algebroid whose anchor is injective on an open dense set is integrable, so $(\Sigma, \pi)$ is an integrable Poisson manifold.

The following result follows from Theorem 5.1.

Theorem 6.3 Two Poisson structures $\pi, \pi^{\prime} \in \mathscr{G}_{n}(\Sigma)$ with the same zero sets $Z(\pi)=Z\left(\pi^{\prime}\right)=$ $\bigsqcup_{i=1}^{n} \gamma_{i}$ and equal modular periods, $T_{\gamma_{i}}(\pi)=T_{\gamma_{i}}\left(\pi^{\prime}\right)$, for $i=1, \ldots, n$, are Morita equivalent.

We now turn our attention to the study of Morita equivalence in $\mathscr{G}\left(S^{2}\right)$. First, we need to collect a few general results on invariants of Morita equivalence.

\subsection{Invariants of Morita equivalence: topology of the leaf space and modular periods}

Let $(M, \pi)$ be a Poisson manifold. Let $L(M)$ be the leaf space of the symplectic foliation of $\pi$, endowed with its quotient topology: for a topological space $X$, a function $f: L(M) \rightarrow X$ is continuous if and only if $f \circ \operatorname{pr}: M \rightarrow X$ is continuous, where $\operatorname{pr}: M \rightarrow L(M)$ is the quotient map.

Let $\left(M_{1}, \pi_{1}\right)$ and $\left(M_{2}, \pi_{2}\right)$ be Poisson manifolds, and let $\left(M_{1}, \pi_{1}\right) \stackrel{J_{1}}{\leftarrow}(S, \Omega) \stackrel{J_{2}}{\rightarrow}\left(M_{2}, \pi_{2}\right)$ be a Morita equivalence bimodule. It is well-known (see e.g. [2,6]) that $S$ induces a bijection of sets $\phi_{S}: L\left(M_{1}\right) \rightarrow L\left(M_{2}\right)$ given by

$$
\phi_{S}(\mathscr{L})=J_{2}\left(J_{1}^{-1}(\mathscr{L})\right), \quad \text { for } \mathscr{L} \in L\left(M_{1}\right) .
$$

The following observation is based on ideas from [12].

Proposition 6.4 The map $\phi_{S}: L\left(M_{1}\right) \rightarrow L\left(M_{2}\right)$ is a homeomorphism of topological spaces.

Proof: Let $F_{i}$ be the subset of $T M_{i}$ consisting of vectors tangent to the symplectic leaves. Let $T J_{i} \subset T S, i=1,2$ be the subbundles tangent to the $J_{i}$-fibers. Then

$$
J_{1}^{*} F_{1}=J_{2}^{*} F_{2}=T J_{1}+T J_{2},
$$

where $J_{i}^{*} F_{i}=\left\{v \in T S \mid T J_{i} v \in F_{i}\right\}$ denotes the pull-back of $F_{i}$. Let $F=J_{1}^{*} F_{1}=J_{2}^{*} F_{2}$. 
Since the fibers of $J_{i}$ are connected, $i=1,2$, the natural maps

$$
\psi_{i}: S / F \rightarrow M_{i} / F_{i}
$$

of leaf spaces are bijections. Moreover, it is not hard to see that $\phi_{S}=\psi_{2} \circ \psi_{1}^{-1}$. So, if we endow $S / F$ with its quotient topology, it is sufficient to prove that $\psi_{i}, i=1,2$, are homeomorphisms.

By the definition of the quotient topology, the map $\psi_{i}: S / F \rightarrow M_{i} / F_{i}$ is continuous if and only if the map $\psi_{i} \circ \mathrm{pr}: S \rightarrow M_{i} / F_{i}$ is continuous (here pr: $S \rightarrow S / F$ is the quotient map). But $\psi_{i}=\operatorname{pr}_{i} \circ J_{i}$, where $\operatorname{pr}_{i}: M_{i} \rightarrow M_{i} / F_{i}$ is the quotient map. Hence $\psi_{i}$ is continuous.

Similarly, $\psi_{i}^{-1}: M_{i} / F_{i} \rightarrow S / F$ is continuous if and only if $\psi_{i}^{-1} \circ \operatorname{pr}_{i}: M_{i} \rightarrow S / F$ is continuous. Since $J_{i}$ is a submersion, this is true if and only if $\psi_{i}^{-1} \circ \operatorname{pr}_{i} \circ J_{i}: S \rightarrow S / F$ is continuous. But $\psi_{i}^{-1} \circ \operatorname{pr}_{i} \circ J_{i}=$ pr. Therefore, $\psi_{i}$ is a homeomorphism for $i=1,2$, which implies that $\phi_{S}$ is a homeomorphism.

The modular vector field and the modular class are well-behaved under Morita equivalence: the bijection of leaf spaces $\phi_{S}$ induced by a Morita equivalence bimodule produces an isomorphism of Poisson cohomologies [17, Thm. 3.1]

$$
\phi_{S}^{*}: H_{\pi}^{1}\left(M_{1}\right) \rightarrow H_{\pi}^{1}\left(M_{2}\right),
$$

which preserves the modular class $[12,16]$,

$$
\phi_{S}^{*}\left(\mu_{\left(M_{1}, \pi_{1}\right)}\right)=\mu_{\left(M_{2}, \pi_{2}\right)} .
$$

We will need the following remark from the construction of the isomorphism (6.1) in [17].

Remark 6.5 Given volume forms $\nu_{1}$ and $\nu_{2}$ on $M_{1}$ and $M_{2}$, respectively, there exists a vector field $X$ on $S$ with the property that $\left(J_{i}\right)_{*} X=X^{\nu_{i}}, i=1,2$. The vector field $X$ is actually hamiltonian, and its Hamiltonian $H$ is determined by the equation

$$
D J_{1}^{*} \nu_{1}= \pm e^{H} J_{2}^{*} \nu_{2}
$$

where $D: \Omega^{k}(M) \rightarrow \Omega^{2 m-k}(M)$ is the symplectic $*$-operator (cf. [3]).

We now observe that the modular periods also behave well under Morita equivalence.

Theorem 6.6 Let $\left(M_{i}, \pi_{i}\right), i=1,2$ be Poisson manifolds, and let $\left(M_{1}, \pi_{1}\right) \stackrel{J_{1}}{\leftarrow}(S, \Omega) \stackrel{J_{2}}{\rightarrow}\left(M_{2}, \pi_{2}\right)$ be a Morita equivalence bimodule. Assume that $Z_{i} \subset M_{i}$ are such that $\left.\pi_{i}\right|_{Z_{i}}=0$ and the isomorphism of leaf spaces satisfies

$$
\phi_{S}\left(Z_{1}\right)=Z_{2}
$$

Let $\Phi_{t}^{i}$ be the flow of the modular vector field $X^{\nu_{i}}$ for some volume form $\nu_{i}$ on $M_{i}, i=1,2$. Assume that $\Phi_{t}^{i}$ takes $Z_{i}$ to $Z_{i}$ for all $t$. Then

$$
\phi_{S} \circ \Phi_{t}^{1}=\Phi_{t}^{2} \circ \phi_{S} \quad \text { in } Z_{1}, \quad \forall t \in \mathbb{R} .
$$

Proof: Let $X$ be a vector field on $S$ such that $\left(J_{i}\right)_{*} X=X_{i}^{\nu_{i}}, i=1,2$ (see Remark 6.5). By the definition of $\phi_{S}$, for each $p_{1} \in Z_{1}$ (which forms by itself a symplectic leaf),

$$
\phi_{S}\left(\left\{p_{1}\right\}\right)=J_{2}\left(J_{1}^{-1}\left(\left\{p_{1}\right\}\right)\right),
$$


which by our assumption on $\phi_{S}$ is a single point $p_{2} \in Z_{2}$. It follows that $J_{1}^{-1}\left(\left\{p_{1}\right\}\right) \subset J_{2}^{-1}\left(\left\{p_{2}\right\}\right)$. Reversing the roles of $p_{1}$ and $p_{2}$, we get $J_{1}^{-1}\left(\left\{p_{1}\right\}\right)=J_{2}^{-1}\left(\left\{p_{2}\right\}\right)$. In particular, it follows that

$$
J_{1}^{-1}\left(Z_{1}\right)=J_{2}^{-1}\left(Z_{2}\right) \text {. }
$$

Thus for any fixed $r \in J_{1}^{-1}\left(\left\{p_{1}\right\}\right)=J_{2}^{-1}\left(\left\{p_{2}\right\}\right)$ we obtain

$$
\Phi_{t}^{2}\left(\phi_{S}\left(\left\{p_{1}\right\}\right)\right)=J_{2}\left(\Phi_{t}(\{r\})\right)=J_{2}\left(J_{1}^{-1}\left(\Phi_{t}^{1}\left(\left\{p_{1}\right\}\right)\right)=\phi_{S}\left(\Phi_{t}^{1}\left(\left\{p_{1}\right\}\right)\right),\right.
$$

where $\Phi_{t}$ is the flow of $X$. Therefore, $\phi_{S} \circ \Phi_{t}^{1}=\Phi_{t}^{2} \circ \phi_{S}$ in $Z_{1}$.

Corollary 6.7 Assume that $\gamma_{i} \subset M_{i}$ are simple closed curves, $U_{i} \supset \gamma_{i}$ are open sets, and that $\left.\pi_{i}\right|_{\gamma_{i}}=0$ and $\left.\pi\right|_{U_{i} \backslash \gamma_{i}} \neq 0, i=1,2$. Assume further that $\left(M, \pi_{1}\right)$ and $\left(M_{2}, \pi_{2}\right)$ are Moritaequivalent via a bimodule $S$. Assume finally that $\phi_{S}\left(\gamma_{1}\right)=\gamma_{2}$. If the modular vector fields are nonzero in $\gamma_{i}, i=1,2$, then

$$
T_{\gamma_{1}}\left(M_{1}, \pi_{1}\right)=T_{\gamma_{2}}\left(M_{2}, \pi_{2}\right) .
$$

Proof: Applying the previous theorem with $Z_{i}=\gamma_{i}, i=1,2$, we obtain that the restriction to $\gamma_{i}$ of the flows $\Phi_{t}^{i}$ of modular vector fields $X_{i}^{\nu_{i}}$ are intertwined by $\phi_{S}$. Thus, for any $p_{1} \in \gamma_{1}$,

$$
\begin{aligned}
T_{\gamma_{1}}\left(M_{1}, \pi_{1}\right) & =\inf \left\{t>0: \Phi_{t}^{1}\left(p_{1}\right)=p_{1}\right\} \\
& =\inf \left\{t>0: \phi_{S}\left(\Phi_{t}^{1}\left(p_{1}\right)\right)=\phi_{S}\left(p_{1}\right)\right\} \\
& =\inf \left\{t>0: \Phi_{t}^{2}\left(\phi_{S}\left(p_{1}\right)\right)=\phi_{S}\left(p_{1}\right)\right\} \\
& =\inf \left\{t>0: \Phi_{t}^{2}\left(p_{2}\right)=p_{2}\right\}, \quad p_{2}=\phi_{S}\left(p_{1}\right) \\
& =T_{\gamma_{2}}\left(M_{2}, \pi_{2}\right) .
\end{aligned}
$$

\subsection{Morita equivalence of topologically stable Poisson structures on $S^{2}$}

We will now show that two Poisson structures in $\mathscr{G}_{n}\left(S^{2}\right)$ are Morita equivalent if and only if they are gauge equivalent up to diffeomorphism. As a result, we find a complete Morita-equivalence invariant for topologically stable Poisson structures on $S^{2}$.

Let $\Sigma=S^{2}$, the 2-sphere. Let $\pi \in \mathscr{G}(\Sigma)$ and write, as before, $\pi=f \cdot \pi_{0}$, where $\pi_{0}$ is a non-degenerate Poisson structure on $\Sigma$ and $f \in C^{\infty}(\Sigma)$. We fix on $\Sigma$ the orientation induced by (the symplectic form corresponding to) $\pi_{0}$. The class of $Z(\pi)$ modulo (orientation-preserving) diffeomorphisms of $\Sigma$ can be described combinatorially by a signed tree in the following way [25, Sec. 2.8]. The vertices of the tree correspond to the connected components of $\Sigma \backslash Z(\pi)$ (i.e., the regions bounded by the curves comprising $Z(\pi))$. The vertex is assigned a positive sign if $f$ is positive on the corresponding region; otherwise the vertex is assigned a negative sign. Two vertices are connected by an edge if and only if the corresponding regions share a boundary. We denote this signed tree by $\operatorname{Tree}(\pi)$.

Lemma 6.8 If two Poisson structures $\pi, \pi^{\prime} \in \mathscr{G}_{n}\left(S^{2}\right)$ are Morita equivalent, then there exists an isomorphism of trees $\phi:$ Tree $(\pi) \rightarrow$ Tree $\left(\pi^{\prime}\right)$, not necessarily preserving signs, so that $T_{\gamma}(\pi)=T_{\phi(\gamma)}\left(\pi^{\prime}\right)$ for every edge $\gamma$ of $\operatorname{Tree}(\pi)$. 
Proof: Assume that $\left(S^{2}, \pi\right)$ and $\left(S^{2}, \pi^{\prime}\right)$ are Morita equivalent. Let $\phi$ be the induced homeomorphism of the leaf spaces of $\left(S^{2}, \pi\right)$ and $\left(S^{2}, \pi^{\prime}\right)$ (see Prop. 6.4).

As a set, the leaf space $L$ of $\left(S^{2}, \pi\right)$ can be identified with the union $Z(\pi) \sqcup\left\{\ell_{1}, \cdots, \ell_{n}\right\}$, where $\ell_{1}, \ldots, \ell_{n}$ are the points corresponding to the 2 -dimensional leaves $\mathcal{L}_{1}, \ldots, \mathcal{L}_{n}$. The quotient topology of $L$ is easily described: the only open subsets of $L$ have the form $U \cup\left\{\ell_{i_{1}}\right\} \cup \cdots \cup\left\{\ell_{i_{k}}\right\}$, where $i_{1}, \ldots, i_{k} \in\{1, \cdots, n\}, k \geq 0$ and $U \subset Z(\pi)$ is an open subset with the property that if $U$ intersects non-trivially a curve $\gamma \subset Z(\pi)$, then for both leaves bounding $\gamma$ the corresponding points of the leaf space occur among $\left\{\ell_{i_{1}}, \ldots, \ell_{i_{k}}\right\}$.

Given $L$ with its topology, consider the collection $\mathcal{Y}$ of all subsets $Y \subset L$ with the property that $L \backslash Y$ is Hausdorff. Order $\mathcal{Y}$ by inclusion. We claim that $X=\left\{\ell_{1}, \ldots, \ell_{n}\right\}$ is a minimal element of $\mathcal{Y}$ of smallest cardinality. First, note that $X \in \mathcal{Y}$, since the relative topology on $L \backslash X=Z(\pi) \subset L$ is Hausdorff. Next, assume that $Y \in \mathcal{Y}$, and $Y \not \supset X$. Then $\ell_{i} \in L \backslash Y$ for some $i \in\{1, \cdots, n\}$. Now all of the points of the boundary of $\mathcal{L}_{i}$ in $S^{2}$ lie in $Z(\pi)$ and cannot be separated from $\ell_{i}$ by open sets; thus all of these points must necessarily be in $Y$. Thus $Y$ must have infinite cardinality.

It follows that $\phi$ must map $X$ to a subset of $L^{\prime}$ with the same minimality property; and hence $\phi$ must take the complement of $X, Z(\pi)$, to $Z\left(\pi^{\prime}\right)$. Thus $\phi$ induces a map between the set of vertices of $\operatorname{Tree}(\pi)$ and $\operatorname{Tree}\left(\pi^{\prime}\right)$.

Now, two vertices $\ell_{i}, \ell_{j} \in \operatorname{Tree}(\pi)$ are connected by an edge if and only if the corresponding regions share a boundary in $S^{2}$. A point $x \in Z(\pi) \subset L$ cannot be separated from $\ell_{k}$ by an open set if and only if $x$ belongs to the boundary of $\mathcal{L}_{k}$ in $S^{2}$. It follows that $\ell_{i}, \ell_{j}$ are connected by an edge if and only if there exists a point $x \in L$, such that $x \neq \ell_{i}, x \neq \ell_{j}$, but which cannot be separated from either of them by an open set. Since $\phi$ is a homeomorphism, it must preserve this property, and thus $\phi$ induces a map of trees from $\operatorname{Tree}(\pi)$ to $\operatorname{Tree}\left(\pi^{\prime}\right)$. The statement about modular periods now follows from Corollary 6.7.

Theorem 6.9 Two Poisson structures $\pi, \pi^{\prime} \in \mathscr{G}_{n}\left(S^{2}\right)$ are Morita equivalent if and only if there exists an isomorphism of trees $\phi:$ Tree $(\pi) \rightarrow$ Tree $\left(\pi^{\prime}\right)$, not necessarily preserving signs, so that $T_{\gamma}(\pi)=T_{\phi(\gamma)}\left(\pi^{\prime}\right)$ for every edge $\gamma$ of $\operatorname{Tree}(\pi)$.

Proof: The necessity follows from Lemma 6.8 .

Assume now that there exists an isomorphism $\phi: \operatorname{Tree}(\pi) \rightarrow \operatorname{Tree}\left(\pi^{\prime}\right)$ satisfying the conditions. Let $\psi: S^{2} \rightarrow S^{2}$ be an orientation-reversing diffeomorphism. By replacing $\pi^{\prime}$ with $\psi_{*} \pi^{\prime}$ (which is obviously Poisson isomorphic, and hence Morita equivalent to, $\pi^{\prime}$ ) if necessary, we may assume that $\phi: \operatorname{Tree}(\pi) \rightarrow \operatorname{Tree}\left(\pi^{\prime}\right)$ is an isomorphism of signed trees.

Choose a function $g \in C^{\infty}\left(S^{2}\right)$ supported on the interior of one of the two-dimensional leaves. Let $\pi^{\prime \prime}=\pi^{\prime}+g \pi$. Since $\pi^{\prime \prime}=\pi^{\prime}$ in a neighborhood of each of the zero curves $\gamma \subset Z(\pi)$, the modular periods of $\pi^{\prime}$ and $\pi^{\prime \prime}$ are equal. Therefore, by Theorem 6.2, $\pi^{\prime}$ and $\pi^{\prime \prime}$ are gauge equivalent up to Poisson diffeomorphism, and hence Morita equivalent (Theorem 5.1) for any such choice of $g$. Also, the isomorphism $\phi$ induces an isomorphism of trees $\phi^{\prime}: \operatorname{Tree}(\pi) \rightarrow$ $\operatorname{Tree}\left(\pi^{\prime \prime}\right)$.

With a suitable choice of $g$, the regularized Liouville volume of $\pi^{\prime \prime}$ can be made equal to that of $\pi$ (see [25] for details). Thus by [25, Thm. 3], $\pi$ and $\pi^{\prime \prime}$ are Poisson isomorphic. We conclude that $\pi$ and $\pi^{\prime \prime}$ are Morita equivalent, and so are $\pi$ and $\pi^{\prime}$, by transitivity of Morita equivalence. 


\section{References}

[1] Bayen, F., Flato, M., Frønsdal, C., Lichnerowicz, A., Sternheimer, D.: Deformation Theory and Quantization. Ann. Phys. 111 (1978), 61-151.

[2] Blaom, A.: A geometric setting for Hamiltonian perturbation theory. Mem. Amer. Math. Soc. 153 (2001), no. 727.

[3] Brylinski, J.-L.: A differential complex for Poisson manifolds. J. Differential Geom. 28.1 (1988), 93-114.

[4] Bursztyn, H.: Semiclassical geometry of quantum line bundles and Morita equivalence of star products. Int. Math. Res. Notices .16 (2002), 821-846.

[5] Bursztyn, H., Waldmann, S.: The characteristic classes of Morita equivalent star products on symplectic manifolds. Comm. Math. Physics 228.1 (2002), 103-121.

[6] Cannas da Silva, A., Weinstein, A.: Geometric models for noncommutative algebras. American Mathematical Society, Providence, RI, 1999.

[7] Cattaneo, A., Felder, G.: Poisson sigma-models and symplectic groupoids. Available at math.SG/0003023.

[8] Charlap, L. S.: Compact flat riemannian manifolds. I. Ann. of Math. (2) 81 (1965), 15-30.

[9] Coste, A., Dazord, P., Weinstein, A.: Groupoïdes symplectiques. In: Publications du Département de Mathématiques. Nouvelle Série. A, Vol. 2, i-ii, 1-62. Univ. Claude-Bernard, Lyon, 1987.

[10] Courant, T.: Dirac manifolds. Trans. Amer. Math. Soc. 319.2 (1990), 631-661.

[11] Courant, T., Weinstein, A.: Beyond Poisson structures. In: Action hamiltoniennes de groupes. Troisième théorème de Lie (Lyon, 1986), 39-49. Hermann, Paris, 1988.

[12] Crainic, M.: Differentiable and algebroid cohomology, van Est isomorphisms, and characteristic classes. Comm. Math. Helv. (to appear). Available at math.DG/0008064.

[13] Crainic, M., Fernandes, R.: Integrability of Lie brackets. Ann. of Math. (to appear). Available at math.DG/0105033.

[14] Dazord, P., Delzant, T.: Le problème général des variables actions-angles. J. Differential Geom. 26.2 (1987), 223-251.

[15] Debord, C.: Groupoïdes d'holonomie de feuilletages singuliers. C. R. Acad. Sci. Paris Sér. I Math. 330.5 (2000), 361-364.

[16] Ginzburg, V. L.: Grothendieck Groups of Poisson Vector Bundles. J. Symplectic Geom. (to appear) . Available at math.DG/0009124.

[17] Ginzburg, V. L., Lu, J. H.: Poisson cohomology of Morita-equivalent Poisson manifolds. Internat. Math. Res. Notices .10 (1992), 199-205.

[18] Gotay, M., Lashof, R., Śniatycki, J., Weinstein, A.: Closed forms on symplectic fibre bundles. Comment. Math. Helv. 58.4 (1983), 617-621.

[19] Hilton, P., Mislin, G., Roitberg, J.: Sphere bundles over spheres and non-cancellation phenomena. In: Symposium on Algebraic Topology (Battelle Seattle Res. Center, Seattle, Wash., 1971), 34-46. Lecture Notes in Math., Vol. 249. Springer, Berlin, 1971.

[20] Jurco, B., Schupp, P., Wess, J.: Noncommutative line bundle and Morita equivalence . Available at hep-th/0106110.

[21] Klimcik, C., Strobl, T.: WZW-Poisson manifolds. FSU-TPI 03/01. Available at math.SG/0104189.

[22] Kontsevich, M.: Deformation Quantization of Poisson Manifolds, I. Available at q-alg/9709040.

[23] Landsman, N.: Quantization as a functor. Available at math-ph/0107023.

[24] PARK, J.-S.: Topological open p-branes. Available at math-ph/0012141.

[25] Radko, O.: A classification of topologically stable Poisson structures on a compact oriented surface. J. Symplectic Geom. (to appear). Available at math.SG/0110304.

[26] Roytenberg, D.: Poisson cohomology of SU(2)-covariant "necklace" Poisson structures on $S^{2}$. J. Nonlinear Math. Physics 9.3 (2002). 
[27] Severa, S., Weinstein, A.: Poisson geometry with a 3-form background. Proceedings of the International Workshop on Noncommutative Geometry and String Theory, Keio University (2001). Available at math.SG/0107133.

[28] Weinstein, A.: The symplectic "category". In: Differential geometric methods in mathematical physics (Clausthal, 1980), 45-51. Springer, Berlin, 1982.

[29] Weinstein, A.: The local structure of Poisson manifolds. J. Differential Geom. 18.3 (1983), 523-557.

[30] Weinstein, A.: Symplectic groupoids and Poisson manifolds. Bull. Amer. Math. Soc. (N.S.) 16.1 (1987), 101-104.

[31] Weinstein, A.: Coisotropic calculus and Poisson groupoids. J. Math. Soc. Japan 40.4 (1988), $705-727$.

[32] Weinstein, A.: The modular automorphism group of a Poisson manifold. J. Geom. Phys. 23.3-4 (1997), 379-394.

[33] Xu, P.: Morita equivalence of Poisson manifolds. Comm. Math. Phys. 142.3 (1991), 493-509. 